\title{
Advanced-Microscopy Techniques for the Characterization of Cellulose Structure and Cellulose-Cellulase Interactions ${ }^{*}$
}

\author{
Jose M. Moran-Mirabal
}

Additional information is available at the end of the chapter

http://dx.doi.org/10.5772/56584

\section{Introduction}

Plants are composed of a variety of different cells with distinct physical characteristics, which are reflected in the different amounts of proteins, lipids, and structural polymers (e.g. polysaccharides and polyphenolic compounds) that are contained within them. One common characteristic to all plant cells is the presence of a rigid cell wall (with thicknesses ranging from 0.1 to $10 \mu \mathrm{m}$ ) that not only conveys to the cell its shape, mechanical strength and pathogen resistance, but also participates in cell-to-cell adhesion, a key interaction that provides plants the robustness that allows them to grow under variable environments. $(1,2)$ The structure of plant cell walls is typically described in terms of three layers: the middle lamella, primary cell wall and secondary cell wall, which have been characterized according to their composition, role during organ growth, and their ability to resist tensile or compressive forces $(1,3)$. Plant cell walls are constructed from a combination of a variety of polysaccharides that can be generally grouped into cellulose, hemicelluloses, and pectic polysaccharides (Figure 1), and whose relative proportions depend on the plant species, specific tissue, and growth stage. Cellulose, the most abundant structural polysaccharide in cell walls (comprising $15-50 \%$ of the dry weight of plant biomass),(3) is a linear polymer of glucose units covalently linked through $\beta(1-4)$ glycosidic bonds. Cellulose glucan chains aggregate through hydrogen bonding and pyranose ring stacking into tightly packed crystalline elementary microfibrils (2-20 nm in diameter), which are the essential scaffold of all plant cell walls. $(2,4,5)$ Hemicelluloses, the second most abundant component of cell walls (10-35\% by dry weight),(3) are highly branched hetero-polymers of pentoses and hexoses that can be broadly classified into xylans, mannans, $\beta$-glucans and xyloglucans. These

\footnotetext{
* Fragments of this chapter have been published in: Moran-Mirabal JM. Study of cellulase-cellulose interactions through fluorescence microscopy techniques. Cellulose. 2013; In Press.
} 


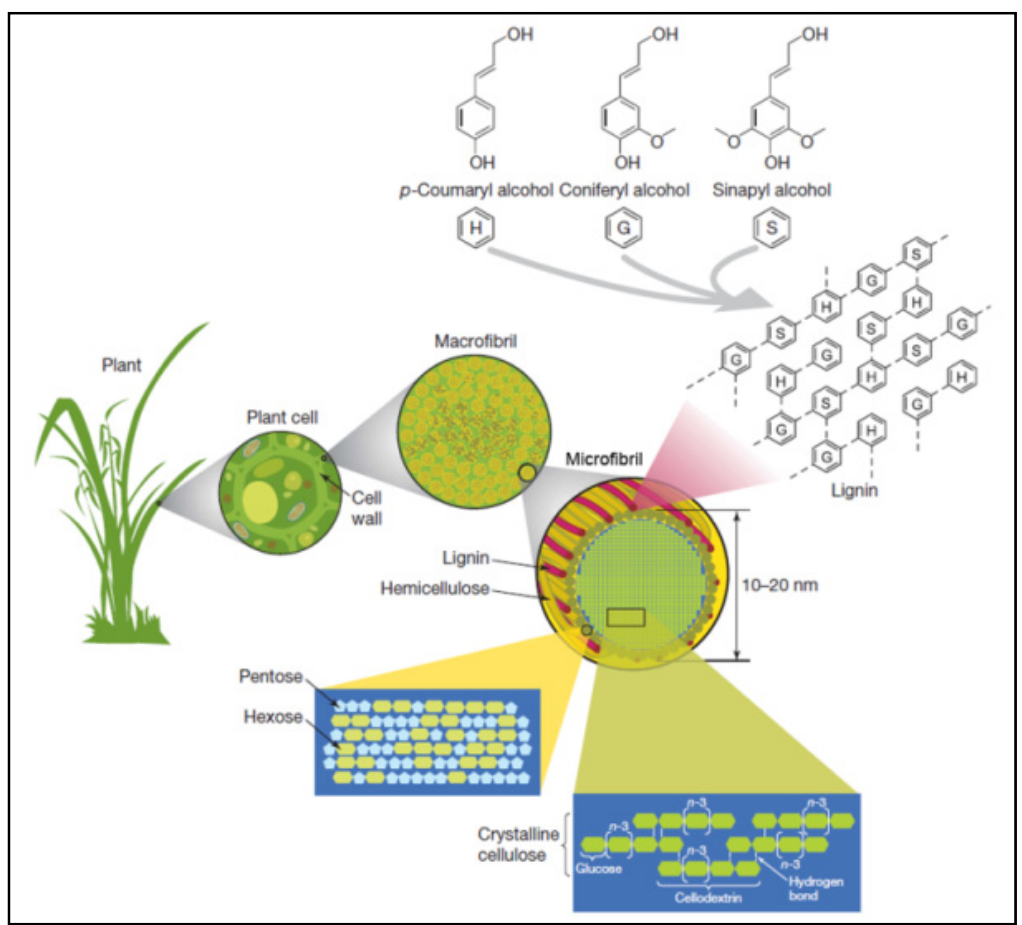

Figure 1. In a simplified representation, the plant cell wall can be visualized as a collection of crystalline cellulose microfibrils sheathed by hemicellulose and lignin. Adapted from (5) with permission from Nature Publishing Group.

branched polysaccharides associate with microfibrils through hydrogen bonds and form, together with pectic polysaccharides a porous matrix (with pores $>10 \mathrm{~nm}$ ) that sheaths the crystalline cellulose core of the microfibrils.(6-8) In woody biomass the cell wall is further reinforced by lignin (5-30\% by dry weight),(3) a three dimensional polymer of phenyl propanoid units that is covalently linked to hemicellulose. The main components that make up lignin polymers are p-coumaryl alcohol, coniferyl alcohol and sinapyl alcohol, the proportional distribution of which varies between plant species and tissue type.(2,5) Lignin acts as nature's glue, forming a protective barrier that limits water and enzyme accessibility to cellulose and gives plants increased resistance to pathogen attack and biomass degradation. The variability in the compositions and relative proportions of structural elements in plant cell walls from different species and tissues results in a high degree of physical and biochemical complexity of plant biomaterials, which creates a challenge for the physicochemical characterization of plant biomass.

Beyond their importance as structural elements in plant morphology and development, the polysaccharides found in the cell wall also represent the major repository of photosynthetically fixed carbon in the biosphere, making them critical for plant, microbial, and animal nutrition and growth, and for the maintenance and balance of Earth's 
ecosystems.(1) Additionally, structural polysaccharides within the cell wall are widely exploited by humans in the elaboration of a myriad of products such as lumber, food, food additives, paper, fibers, textiles, biocomposites, bioplastics, and biofuels. All these products and their characteristics depend intimately on the composition and structural arrangement of cell walls. In particular, due to their importance in the production of biofuels and bioproducts from plant materials, much recent research has focused on studying the changes effected by thermal, chemical and biochemical processes on the structural arrangement of cellulose, hemicellulose and lignin.

Traditionally, cellulose structure has been studied at high resolution through scanning electron microscopy $(7,9-14)$ or transmission electron microscopy.(9,14-20) In these techniques, cellulose samples must be prepared through drying, coating or staining methods that render the samples compatible with electron microscopy. Despite the exquisite detail that can be gained from these imaging techniques and the wealth of knowledge that has already been obtained through them, the sample preparation procedures can introduce artefacts through modifications to the physical structure of cellulose, such as the collapse of the micro- and nanoporous structure of plant-derived cellulose, the recrystallization and aggregation of elementary microfibrils, or by disrupting the biomolecular interactions occurring at the interface of the insoluble cellulose, such as the dislodgement of proteins weakly adsorbed to the surface of cellulose fibrils. This has resulted in a search for experimental techniques that can reveal the structure of cell wall components with high spatial resolution under environmental conditions that do not modify the structure or the biomolecular interactions. Furthermore, techniques able to visualize the structural and compositional changes that occur due to the biochemical deconstruction of plant material in real time and at temperatures relevant for industrial processing could offer insights into the limitations of biomass conversion processes. This chapter explores a set of advancedmicroscopy techniques that allow imaging of cellulosic structures at high resolution under hydrated conditions and how they have been applied to study cellulose and the biomolecular interactions that occur with cell-wall degrading enzymes. The chapter will end with an overview of the possibilities that have opened over the past years due to the development of these novel imaging approaches.

\section{Advanced-microscopy techniques}

\subsection{What can advanced-microscopy offer for the study of cellulose?}

The term advanced-microscopy refers in general to the host of techniques that allow us to visualize biological samples with resolution in the submicron to nanometer scale. This level of detail enables the study of biomolecular structures, interactions, and mechanisms of catalysis at the fundamental length scale of proteins, lipids, amino acids, glycans, and other biomolecules. In particular, for the study of cellulosic materials this implies the ability to visualize the arrangement of cellulose from the length scale of the individual glucan chains (sub-nanometer) to that of their aggregates in the form of elementary microfibrils (nanometers) or cell wall macrofibrils (microns). Furthermore, the study of the interactions 
between cell wall modifying enzymes and cellulosic structures requires the ability to identify individual proteins, their localization, dynamic motions, conformation, and catalytic activities, as well as the changes that they effect on the structural arrangement of cellulose. Ideally, all these measurements would be obtained under experimental conditions that preserve the cellulosic materials in their native state, maintain the full functionality of cell-wall modifying enzymes, and also provide high spatial and temporal resolution to observe any dynamic restructuring and biomolecular interactions in real time. It is clear that a single technique cannot provide all this information simultaneously with the optimal temporal and spatial resolution, and that complementary techniques are needed to achieve a full understanding of the structural and biochemical changes that occur in cellulosic materials as they undergo physical, chemical or biochemical processing.

A number of techniques, including certain types of electron microscopy (EM), scanning probe microscopy (SPM), and optical microscopy can be considered advanced-microscopy techniques because they can yield images with spatial resolution ranging from the micrometer to the sub-nanometer scale. Each one of these types of microscopy has its advantages and limitations in their application to the study of cellulose: EM techniques can yield exquisite detail even at the sub-nanometer scale, but in most cases are limited to dry samples that must ideally be rendered conductive to make them compatible with EM imaging; SPM techniques have the ability to image immobile substrates with sub-nanometer resolution, but must come into contact or be in very close proximity with the sample, cannot effectively image large surface areas, and cannot track multiple biomolecular species simultaneously; and optical microscopy can track multiple biomolecular species with high temporal resolution in large surface areas, but is limited in spatial resolution by the diffraction of light through the optical microscope, as well as by the refraction and scattering of light in thick biological samples. Thus, the choice of experimental advanced-microscopy techniques to image a cellulosic sample depends strongly on the information sought, the complexity of the system, the environmental requirements, and the spatial and temporal resolution desired. In this chapter we focus on a subset of advanced-microscopy techniques which yield images with micron to nanometer scale spatial resolution over periods ranging from seconds to hours, while allowing experimental conditions that provide appropriate temperatures for the catalytic activity of enzymes and keep the samples hydrated in solutions with biologically relevant ionic strength and $\mathrm{pH}$. These capabilities make them ideally suited for the study of cellulose and the interaction between cell wall degrading enzymes and cellulosic substrates.

The discussion throughout the chapter will center on recently developed SPM and fluorescence microscopy techniques, which have been applied to the visualization of the structural arrangement of cellulose and the interactions that occur at the interface between insoluble cellulosic materials and enzymes, with sub-micron to nanometer scale resolution. These techniques have made it possible to study the structural arrangement of cellulose microfibrils and elementary glucan chains, including their intermolecular spacing, cellobiose unit repeat and individual glucose unit distances, both for dry and hydrated cellulosic materials. Furthermore, the use of fast-acquisition SPM and fluorescence techniques has enabled the observation of the binding and molecular motion of individual or multiple cell- 
wall modifying enzymes and their corresponding binding and catalytic modules even at the single molecule level. These advanced-microscopy techniques have also been recently applied in the study of the depolymerization of cellulose by individual cell wall modifying enzymes and by commercially available cellulolytic enzyme cocktails. The use of advanced-microscopy techniques for the study of cellulose, especially in the complex environment of the cell wall, is still in its infancy and many opportunities exist for the fundamental study of cell wall structure, enzyme-cellulose interactions, and for the study of the changes that occur in cellulose structure and biochemical composition as cell walls are deconstructed through different treatments used in industrial settings. As will be seen in the discussion to follow, the development of new SPM and fluorescence microscopy approaches has made it possible to study cellulose with high spatial and temporal resolution under conditions that are biologically relevant. These studies will surely lead to an improved understanding of the organization of the cell wall and of the way cellulolytic enzymes operate. This in turn can steer the development of deconstruction technologies that more efficiently convert plant biomass into useful raw materials for the manufacturing of renewable and sustainable products.

\subsection{Scanning Probe Microscopy}

Scanning Probe Microscopy techniques (SPM) measure the interaction between the sample and a small sample probe, which is brought into contact or very close proximity to it. An image of the sample under investigation is then constructed by scanning the tip and collecting pixel by pixel data, which can then be displayed as a three dimensional reconstruction. SPM techniques allow the direct characterization of the surface of biological specimens with very high spatial resolution and with very little sample preparation.(4) Furthermore, SPM techniques allow imaging biological samples under dry or aqueous conditions and with suitable chambers that can provide temperature-controlled environments. Thus, they are ideal for the structural and biochemical characterization of cellulosic materials and for the study of the interactions between cell-wall degrading enzymes and the surface of insoluble cellulose substrates.

Atomic Force Microscopy (AFM) is a high-resolution SPM technique, which has become one of the most widely utilized tools in real-time imaging of biological samples due to its ability to record surface topography and properties at the nanoscale. The basis of AFM operation is quite simple and lies in the interaction between a sharp tip located at the end of a microfabricated cantilever and the sample surface.(21) The tip, with a radius ranging from of a few nanometers (silicon-based tips) to a few angstroms (carbon nanotube tips), senses a distance-dependent potential that is the result of a multitude of interactions with the surface, including van der Waals, chemical, electrostatic, capillary, and magnetic forces, among others.(22) As the tip is brought into close proximity with the surface, these intermolecular forces deflect the cantilever. The deflection is measured by tracking the displacement of a laser spot that is reflected off the back of the cantilever and imaged onto a photodiode array (Figure 2). The tip can be raster-scanned across the sample using a piezoelectric stage, with the recorded cantilever deflection as a function of position yielding a surface map of the biological sample. The resolution of the surface map is limited by the 


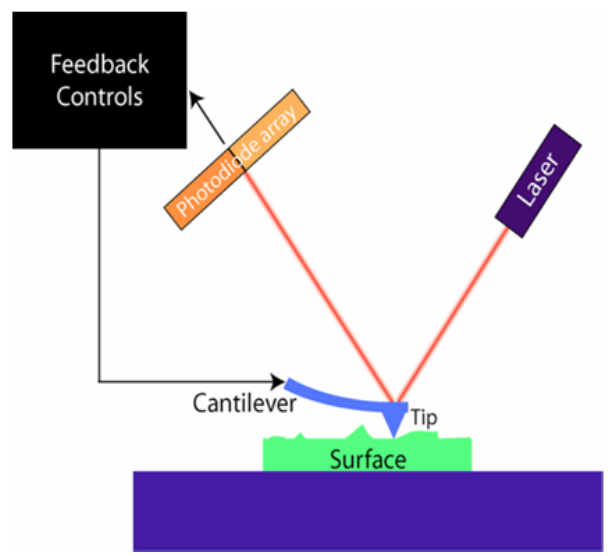

Figure 2. Schematic depiction of a typical atomic force microscope setup. As the tip is brought into close proximity of the surface the cantilever is deflected by attractive or repulsive forces. The displacement is monitored using a laser spot bounced off the back of the cantilever and imaged on a photodiode array. Feedback controls maintain a constant force on the tip.

size of the AFM tip used, and under optimal conditions can reach $0.1 \mathrm{~nm}$ in the vertical and $0.5 \mathrm{~nm}$ in the lateral directions.(21) In a typical AFM setup, the tip-to-substrate distance is controlled by a feedback mechanism, which adjusts the tip position to maintain a constant force between the tip and the sample. For imaging of soft biological samples, the force applied by the cantilever is usually limited to the range between 50 and $100 \mathrm{pN}$ to minimize sample deformation and avoid the disruption of biomolecular interactions (21).

AFM scanning can be performed in two modes: contact or tapping. In static or contact mode, AFM imaging is performed in contact with the sample, where the overall force is repulsive. The main drawback of contact mode for imaging biological samples is that the forces applied during tip scanning can sweep away weakly bound objects or deform soft samples. On the other hand, tapping mode is achieved by driving the cantilever into oscillation at its resonant frequency and allowing limited interaction between sample and tip. The amplitude, phase and frequency of the oscillations are changed by the tip-sample interactions, leading to a description of the surface characteristics. Nowadays, amplitude modulation is the most widely used non-contact mode. In this mode, changes in the oscillation amplitude and phase provide the feedback for imaging.(23) Amplitude variations allow the mapping of the surface topography, while changes in phase allow the discrimination between different types of materials. Surface topography, rigidity, viscosity, as well as adhesion forces can thus be studied through non-contact AFM.

Until recently, one of the main limitations to AFM was that the acquisition of a single image of a 1-micron square area would take several seconds, which precluded the use of AFM to study molecular motions that occur within the millisecond time regime. Efforts towards realizing high speed AFM were pioneered in the 1990s and by 2001 a complete system for the fast acquisition of high quality images was reported. This system introduced small cantilevers with high resonant frequencies $(\sim 600 \mathrm{kHz}$ in water) and a suitable optical 
detection system, which allowed acquisition times of $80 \mathrm{~ms}$ per frame. Later improvements in the z-scanner damping system and the feedback loop increased sample stability and minimized the tip-sample interactions, enabling acquisition times as fast as $30 \mathrm{~ms}$ per frame.(24) Nowadays, the most advanced tapping-mode, high-speed AFMs are being utilized to observe the motions of biomolecular motors such as kinesin, myosin $\mathrm{V}$, and dynein, $(25,26)$ as well as the motions of processive cell wall degrading enzymes. $(27,28)$ The ability to monitor surface topography changes along with biomolecular interactions in real time under aqueous environments and under controlled temperature, $\mathrm{pH}$ and ionic strength conditions make AFM a very useful tool for the study of cellulose, its structural properties, and the interactions that occur between insoluble cellulosic substrates and cell wall degrading enzymes.

\subsection{High-resolution fluorescence microscopy}

Fluorescence microscopy has become one of the most utilized techniques for the study of biological systems for its high sensitivity, selectivity and the ability to monitor multiple species under biologically relevant conditions. Fluorescence arises from the spontaneous emission of a photon from a molecule (fluorophore) in which the absorption of an incident photon causes an electron to transition to an excited singlet state and this electron subsequently transitions to the ground state via the emission of a second, lower energy, photon.(29) Fluorophores that emit in the visible wavelength range are usually molecules with multiple aromatic rings, such as the dyes from the cyanine, fluorescein and rhodamine families or fluorescent proteins like those derived from the green, yellow, red, and cyan fluorescent proteins. Commercially available organic fluorophores are further modified with reactive groups that allow the covalent attachment of the organic dyes to thiol, amine, alkyne, and hydroxyl groups, among others. Conversely, fluorescent proteins can be expressed within the target system as proteins fused to the targeted protein or peptides, which allows fluorescent tagging in vivo.(30-32) The ability to easily tag proteins, lipids, nucleic acids or glycans with fluorescent moieties allows the selective observation of these biomolecules over the background of the biological system of interest. Furthermore, through the use of high performance optics and very sensitive detectors it is possible to observe the fluorescence emitted from the tagged biomolecules, enabling the study of biomolecular localization, transport, binding, catalysis, and other biomolecular interactions even down to the level of single molecules. A number of microscopy techniques have been developed to exploit the advantages of fluorescence emission to study these biomolecular interactions, including confocal fluorescence microscopy, total internal reflection fluorescence microscopy, fluorescence correlation spectroscopy, Förster resonance energy transfer, single molecule tracking, and super-resolution fluorescence microscopy, among others. Through these techniques researchers aim at maximizing the fluorescence photons collected while minimizing the background noise to enhance the sensitivity and the spatio-temporal resolution of the measurements.

The implementation of fluorescence microscopy can be done in different configurations, all of which aim at achieving the highest fluorescence signal to background noise possible. All 
(a)

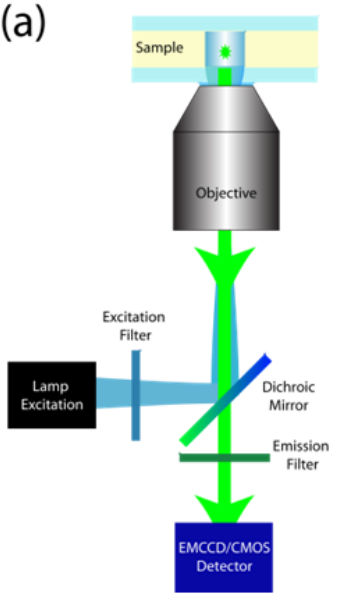

(b)

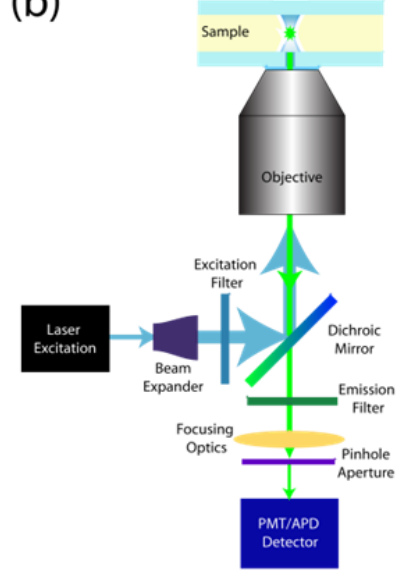

(c)

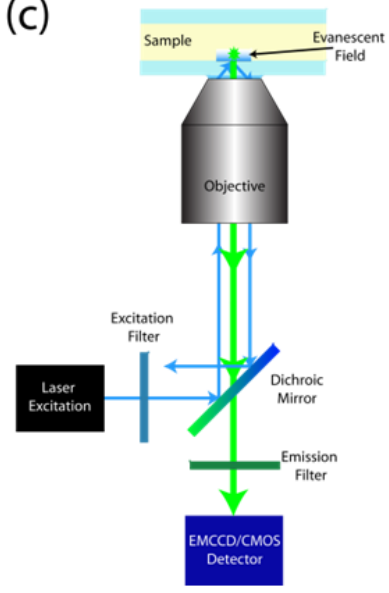

Figure 3. All configurations for fluorescence microscopy imaging share common basic elements: excitation source, excitation and collection optics, and detectors. (a) Epifluorescence microscopy utilizes a broad lamp excitation source for widefield illumination and a high-sensitivity camera for collection. (b) Scanned Confocal and Stimulated Emission-Depletion Super-Resolution microscopy utilize a laser excitation source for point illumination and PMT or APD for collection. (c) Total Internal Reflection and photo-switching based Super-Resolution microscopy utilize a laser excitation source for widefield illumination and a high-sensitivity camera for collection. Adapted from Reference (101).

these experimental configurations share some basic constitutive elements: an excitation source, which can be mono chromatic or wide spectrum and coherent or incoherent; optics for excitation and collection, including excitation and emission filters, dichroic mirrors, and polarizing optics, among others; and one or multiple highly sensitive detectors, such as avalanche photodiodes, photomultiplier tubes, and EMCCD or CMOS cameras (Figure 3). The available microscope configurations can be broadly divided into two main groups: those that detect the emitted fluorescence in wide-field mode (e.g. epifluorescence, TIRF, and photo-switching type Super Resolution microscopy) and those use point detection (e.g. laser scanning confocal microscopy and Stimulated Emission-Depletion Super Resolution microscopy). The selection of the configuration to be used depends on the observables to be measured, the sample and sample requirements, and the spatial and temporal resolution desired. As mentioned before, no single technique can yield all the measurements simultaneously with the maximum spatial and temporal resolution. Thus the selection of the appropriate fluorescence technique is crucial to the successful, reliable and reproducible measurement of sample properties and biomolecular interactions. Below, a few configurations and experimental techniques that have been applied to the study of cellulose or that hold great promise for it are discussed in detail.

Epifluorescence is perhaps the simplest implementation of fluorescence microscopy, but also the most versatile and widely used. The configuration for epifluorescence (Figure 3-a) consists of a broad spectrum light source, such as a mercury arc lap or a metal halide lamp, which emits light in the visible spectrum with enough intensity to excite fluorescence from 
the specimen; a set of excitation and emission optics, which include an excitation filter that selects the band of wavelengths appropriate for excitation of the fluorophore, a dichroic or dichromatic mirror that selectively transmits or reflects wavelengths of incident light, allowing the separation of excitation and emission photons for the orthogonal observation of fluorescence, and an emission filter with a transmission band for wavelengths of light that separates the reflected and scattered light from fluorescence emitted from the specimen; and a sensitive imaging device, such as an electron multiplication charge-coupled device (EMCCD) or a complementary metal-oxide semiconductor (CMOS) camera, which collects the photons at the image plane of the microscope and translates them into an electronic signal that can be recorded in the form of a picture where the pixel intensity is a reflection of the fluorescence emitted from the sample. The configuration for epifluorescence microscopy relies on the excitation and the collection of the emitted light through a high quality objective with magnifications ranging from 2 to $100 \times$, and numerical apertures as high as 1.49 , which allow the efficient collection of photons without chromatic or spherical aberrations. Through this configuration it is possible to image samples containing multiple fluorophores with temporal resolution ranging from milliseconds (EMCCD) to microseconds (CMOS). The use of appropriate calibration standards also allows the use of epifluorescence for the quantitative characterization of concentrations of biomolecules within biological samples. Furthermore, different microscope configurations allow the use of environmental chambers to provide capabilities for temperature control, perfusion of fluids and gases, and integration of electrical measurements, among others. The main limitation to epifluorescence is its poor spatial resolution, which is limited by the diffraction and scattering of light as it travels from the sample to the collection objective and through the optical components of the microscope. Rayleigh's criterion defines the highest achievable resolution in the transverse sample plane:

$$
r_{x-y}=\frac{0.61 \lambda}{N A}
$$

where $\lambda$ is the wavelength of the emitted light and NA is the numerical aperture of the objective, whereas the axial resolution is typically two to three orders of magnitude larger. As an example, a fluorescent sample emitting at $525 \mathrm{~nm}$, observed with a 60x/1.4 NA objective, would be observed with a maximum resolution in the transverse plane of $\sim 230$ $\mathrm{nm}$. Because of its versatility, quantitative epifluorescence has been widely used to visualize a wide range of biological systems. The development of more sensitive cameras, more intense illumination sources and better optics will undoubtedly keep quantitative epifluorescence microscopy as a versatile tool for the study of biological structure and the characterization of biomolecular interactions.

Confocal microscopy improves on the axial resolution of the fluorescence microscope and allows imaging three-dimensional structures through optical sectioning. In the configuration for laser scanning confocal microscopy (Figure 3-b) a collimated laser is used as an excitation source to overfill the back aperture of a high-NA objective, which focuses light into a diffraction-limited spot at the focal plane. The same objective collects the fluorescence emitted from the sample, which is filtered through the dichroic mirror and emission filter, 
and focuses it onto a small pinhole aperture. This aperture is placed at the conjugate focal plane of the objective and serves to reject out-of-focus light. Typically, the confocal aperture diameter is selected such that it is slightly larger than the transverse dimension of the illumination volume (Eq. 1) multiplied by the objective magnification. (33-35) The spatial filtering provided by the confocal aperture improves the axial resolution of the fluorescence microscope by collecting emitted fluorescence light only from the focal plane. The size of the observation volume depends on the wavelength of the emitted light $(\lambda)$, the objective's $N A$ and the index of refraction of the sample medium $(n)$. Equation 1 defines the transverse dimensions of the observation volume, while the axial dimension is given by

$$
\mathrm{r}_{\mathrm{z}}=\frac{2 \mathrm{n} \lambda}{\mathrm{NA}^{2}}
$$

From these, the confocal observation volume can be calculated as the volume of an ellipsoid. Following the same example as above, the axial resolution for the observation of a sample in aqueous medium $(n=1.33)$ with a confocal setup would be $\sim 710 \mathrm{~nm}$, which yields an observation volume of approximately $0.1 \mathrm{fL}$. After the light passes through the confocal aperture, it is collected by a high-sensitivity photon detector, such as an avalanche photodiode (APD) or a photomultiplier tube (PMT). These detectors convert incident photons into electronic signals that can be recorded. Because the signals recorded from the illuminated focal volume represent a single pixel of information, two-dimensional images can be reconstructed by scanning the illumination across the sample in the $x$ and $y$ planes. Scanning can also be extended to the axial dimension by changing the focal plane, allowing the collection of multiple optical z-sections (z-stacking). This enables the reconstruction of the fluorescence profile arising from complex three-dimensional structures with improved resolution and signal to noise ratio. The main drawback of scanning confocal microscopy is that the collection of a 2D image can take several seconds, while a 3D stack can take up to several minutes, depending on the number of optical sections acquired. Thus, dynamic events that are in the sub-second time regime cannot be observed through confocal microscopy. Yet, the improved spatial resolution and the ability to collect fluorescence in multiple channels simultaneously have made confocal microscopy a widely used technique in the biological sciences, and a good candidate for the study of cellulosic samples with complex structure.

Total Internal Reflection Fluorescence Microscopy (TIRFM) is another configuration, which is well suited to study molecular phenomena because it can selectively excite fluorescence from a thin sample layer adjacent to a glass surface. In TIRFM, the incidence of the excitation laser beam upon the interface between a high and a low index of refraction material (typically a glass-water interface) at an angle beyond the critical angle results in TIR of the incident light. Although under TIR no light propagates through the interface, an exponentially decaying (evanescent) electromagnetic field penetrates the sample. This evanescent field is capable of exciting fluorescence emission from fluorophores located near the interface. The penetrating depth of this evanescent field depends on the incident angle and is usually in the $50-250 \mathrm{~nm}$ range. Due to the nature of the evanescent field, the excitation volume is large in the transverse dimensions but highly confined in the axial 
dimension, which greatly reduces background fluorescence from out-of-focus planes and results in images with very high signal-to-noise ratio. TIRFM imaging can be done in two common configurations: prism-based (36) and objective-based.(37-39) In prism-based TIRFM, the laser beam is coupled into the sample through a trapezoidal or cylindrical prism using an index-matching medium (e.g. glycerol or oil). TIR is achieved by coupling the illumination at angles beyond the critical angle and the microscope objective is used to collect the photons emitted by the sample, which are filtered by the dichroic mirror and the emission filter. Thus, the fluorescent sample is imaged from the other side of the specimen using a high-sensitivity camera, which captures the emitted fluorescence. An advantage of this configuration is the low cost of the components, although the incidence angles are restricted to a few discrete values defined by the prism characteristics. Objective-based TIRFM, on the other hand, couples the laser beam via the same objective used for imaging (Figure 3-c). Through the use of a moving lens, the position of the laser beam can be translated axially from the center to the edge of the back aperture of the objective, causing the incident light to hit the glass/sample interface at an angle. This has become the most popular configuration because it allows a continuous range of incidence angles, with a maximum angle determined by the objective's $N A$. By using a high $N A$ objective, supercritical illumination angles can be achieved that are suitable for TIRFM imaging of dense biological samples such as cells or cellulose. The fluorescence emitted from the sample can then be imaged through the same objective onto a high-sensitivity camera (e.g. EMCCD or CMOS). Key advantages of through-the-objective TIRF are that the use of highmagnification, high-NA objectives allows improved lateral resolution, that the photons have a shorter path to the collection optics, that the top of the sample can be open and easily accessible, and that switching between epifluorescence and TIRF can be achieved easily by changing the position of the incident beam. In addition, this configuration allows illumination with multiple laser wavelengths either simultaneously or in rapid succession for imaging of multiple fluorophores. Several excellent reviews of the implementation of TIRF and the comparison between the experimental configurations have been published.(36-41)

A number of fluorescence microscopy techniques have been developed over the past decades to take full advantage of the capabilities of the microscope configurations described above in order to measure specific observables with the highest spatial and temporal resolution. These techniques can be implemented using different microscope configurations, depending on the sample characteristics and the sensitivity desired. One such technique is fluorescence recovery after photobleaching (FRAP), which can be implemented in epifluorescence, confocal, or TIRFM setups. First described by Axelrod in 1976,(42) FRAP is a method for measuring the two-dimensional lateral mobility of fluorescent particles or molecules. In this method, a small spot of a fluorescent sample is permanently photobleached through exposure to a high intensity illumination source, such as an arc lamp or a laser beam. Subsequently, imaging of the sample with the same illumination source under attenuated intensity monitors the recovery of the fluorescence due to molecular transport. With this technique it is possible to identify the type of transport process present in the sample (e.g. diffusion, restricted diffusion, flow), measure the lateral 
diffusion coefficient of the fluorescent species, and identify the fraction of the fluorescent molecules that are mobile within the sample. More recently, models have also been developed to fit FRAP data from systems where binding occurs at the same time scale as the transport phenomenon of interest. $(43,44)$ This has enabled the study of complex biological environments, such as the cytoplasm, where the molecular association to immobile structures hinders the diffusion of the molecular species of interest.(45-49) As will be discussed below, FRAP has already been used to measure the mobility of cellulases on cellulosic substrates.

Förster Resonance Energy Transfer (FRET) is a molecular process that decreases the photon output from fluorophores, a fact that has been tuned into a useful technique to measure molecular distances arising from the close association of two biomolecules or from the mobility of fragments within a single biomolecule. FRET occurs when the emission spectrum of a fluorophore, the donor, overlaps with the absorption spectrum of a second molecule, the acceptor.(50) The basis of FRET lies in the non-radiative transfer of energy from the donor to the acceptor vial long-range dipole-dipole coupling.(51) The energy transfer between the donor and the acceptor depends both on the extent of the spectral overlap and, due to dipole coupling, the distance between them. The FRET efficiency of a donor-acceptor pair is defined as:

$$
\mathrm{E}=\frac{\mathrm{R}_{0}^{6}}{\mathrm{R}_{0}^{6}+\mathrm{r}^{6}}
$$

where $r$ is the distance between donor and acceptor pair and $R_{0}$ is the characteristic Förster radius (FRET efficiency of 50\%) for the pair. In the practical implementation of FRET where the donor and acceptor are both fluorescent molecules, the sample is illuminated with an excitation source matched to the donor's absorption band. If the FRET pair is in close proximity, energy transfer will occur between the donor and the acceptor, resulting in the excitation and subsequent emission of fluorescence from the acceptor fluorophore. The fluorescence emitted is recorded in both the donor and acceptor channels and the FRET efficiency $(E)$ can be calculated. Because $E$ depends strongly on the separation between donor and acceptor, the ratiometric measurement of fluorescence emission from the pair can be effectively used as a spectroscopic molecular ruler.(50) Typical values of $R_{0}$ for fluorescent FRET pairs lie in the 20-100 A range and optimal sensitivity of donor quenching is achieved at distances where $0.7 \mathrm{R}_{0}<\mathrm{r}<1.5 \mathrm{R}_{0}$.(52) Experimentally, FRET can be done in two modalities: through widefield imaging of molecules immobilized on a solid surface or through point probing of molecules in solution. FRET has been used in a wide range of applications, even at the single molecule level, and a number of excellent reviews exist on the theory and applications of this technique.(51,53-56)

Over the past decades, the development of highly sensitive cameras and bright, stable fluorescent dyes has allowed life scientists to image single molecules (SMs) through fluorescence microscopy. The ability to visualize and track individual fluorescent molecules over time permits a more direct characterization of their dynamic behaviour, including molecular association, molecular transport, conformational changes, and catalytic activities. 
Furthermore, the study of SMs can evidence heterogeneous behaviour, or help identify rare molecular subpopulations that would otherwise be averaged and masked by ensemble techniques. The downside of SM experimentation is that in order to get statistically significant measurements, large data sets are required to construct histograms that yield discrete values of the experimental observables. Yet, the information obtained through SM experimentation has proven very valuable and the use of SM techniques has increased exponentially over the last decade. In fluorescence microscopy, SM experimentation is performed in two modes: point detection and widefield imaging. In point detection mode, a laser illumination beam is focused into a diffraction-limited excitation volume, which limits the background fluorescence from out-of-focus planes and gives a very high signal-to-noise ratio. As fluorescent molecules are transported through the excitation volume, they emit a fluorescent signature that is collected and recorded by a highly sensitive detector, such as a PMT or an APD. This fluorescence signature, in the form of bursts of recorded photons, can be analyzed through the photon counting histogram $(\mathrm{PCH})(57-60)$ or fluorescence correlation/crosscorrelation spectroscopy (FCS/FCCS) techniques.(61-67) In this way, the collection of fluorescence photon bursts allows the study of dynamic molecular behaviour, such as diffusion, transport, association and conformational changes, with high temporal resolution ranging from the nanosecond to the second time scale. The strength of point detection SM experimentation is the high temporal resolution afforded by the detectors used, with the caveats of having to measure one molecule at a time and that molecular trajectories can only be followed within the focused excitation volume. On the other hand, widefield SM techniques use configurations such as those for epifluorescence or TIRF microscopy to illuminate a sample containing a sparse collection of fluorescent molecules, and SM images are collected using high sensitivity EMCCD or CMOS cameras. In this modality, the field of view can cover areas of hundreds to thousands of square microns where hundreds of molecules can be visualized at a time. This allows the simultaneous collection of SM data from many individual molecules, reducing the number of experiments required to obtain the required measurements for statistical significance. The main limitations of widefield SM techniques are that the molecules can only be followed for a period of time determined by photobleaching and that the temporal resolution is limited by time it takes the camera to acquire the image (typically in the millisecond range). In the analysis of widefield SM images, each molecule within the image is identified and localized through algorithms that fit the SM emission profile to a theoretical point spread function, such as a two-dimensional Gaussian or a Bessel function.(68-70) This results in the ability to pinpoint the location of individual molecules with precisions that are well below the diffraction limit. Experimentally, the number of photons collected for each molecule and the background determine the accuracy of localization(71,72) and values as low as one nanometer have been reported in the literature.(73) Furthermore, the collection of sequential images and their analysis with SM tracking (SMT) algorithms allows tracking the active or passive transport of individual molecules in real time. Thus, through widefield SM techniques it is not only possible to pinpoint the location of individual molecules and their association kinetics, but it is also possible to reconstruct their molecular trajectories. The simultaneous acquisition of SM images from two spectrally distinct channels also permits the extension of widefield SM 
experimentation to other techniques, such as SM colocalization and SMFRET. SM fluorescence techniques have gained widespread acceptance and are being used for the study of numerous biological systems. The interested reader is referred to the wealth of SM fluorescence reviews for additional information on the different implementations, applications, and the limitations for these advanced-microscopy techniques.(74-86)

Another advanced-microscopy technique that is suitable for the study of cellulose structure and its temporal evolution as a result of physical, chemical or biochemical treatments is super-resolution fluorescence microscopy (SRFM). The term SRFM has been applied to a host of techniques which all aim at obtaining sub-diffraction resolution images from macromolecular assemblies. These assemblies typically contain fluorescently labeled molecules that can be selectively excited to emit photons, and their emission profile can be localized within the field of view with high precision in a manner analogous to the SM localization and tracking approaches described above. SRFM includes methodologies that take advantage of nonlinear optical effects to condition or reduce the size of the excitation point spread function (Stimulated Emission-Depletion or STED, Saturated Structured Illumination microscopy, SSIM)(87-91), or that reconstruct super-resolution images from the emission profiles of individual fluorescent molecules that are selectively (Photo-Activated Localization Microscopy or PALM, and related techniques)(92,93) or randomly (Stochastic Optical Reconstruction Microscopy, STORM, blink microscopy or dSTORM)(94-98) switched to emit fluorescence. While all these approaches obtain similar resolution $(<50$ $\mathrm{nm}$ ), they have different attributes and equipment requirements that make them more or less amenable to implementation, and a number of reviews exist that compare them. $(88,99,100)$ For example, photoswitching techniques allow a facile implementation, without the need for expensive instrumentation, and shift the focus of the SRFM technique development from hardware to the dyes utilized for imaging. Recently, Tinnefeld and collaborators have reported the ability to perform SRFM with conventional fluorescent dyes, provided that suitable imaging wavelength intensities and aqueous medium can be utilized.(95-97) This imaging medium can be utilized with protein or nucleic acid systems, and could be well suited for imaging of cellulose-protein interactions.(101) Despite the difference in operational mechanisms, the principle behind SRFM techniques (Figure 4) is that in a sample that contains a large number of fluorescent molecules, under appropriate experimental conditions, only a few of them are conditioned to emit fluorescence at any given time. The fact that only a sparse collection of molecules is imaged enables their localization with an accuracy that is only limited by the number of photons collected for each emitter. Thus, by continuously illuminating the specimen, and imaging sparse collections of fluorescent molecules in each frame, their position can be determined with great precision, and a final image of the macromolecular structure can be reconstructed. Using these SRFM imaging approaches, the structural arrangement of cellulose microfibrils within a host of substrates could be reconstructed from the aggregated information of fluorescent cellulose-binding proteins. This information could be obtained in real time and with a spatial resolution that can reach the fundamental length scale of proteins $(5-20 \mathrm{~nm})$ and elementary cellulose microfibrils (widths of 5-30 nm). 

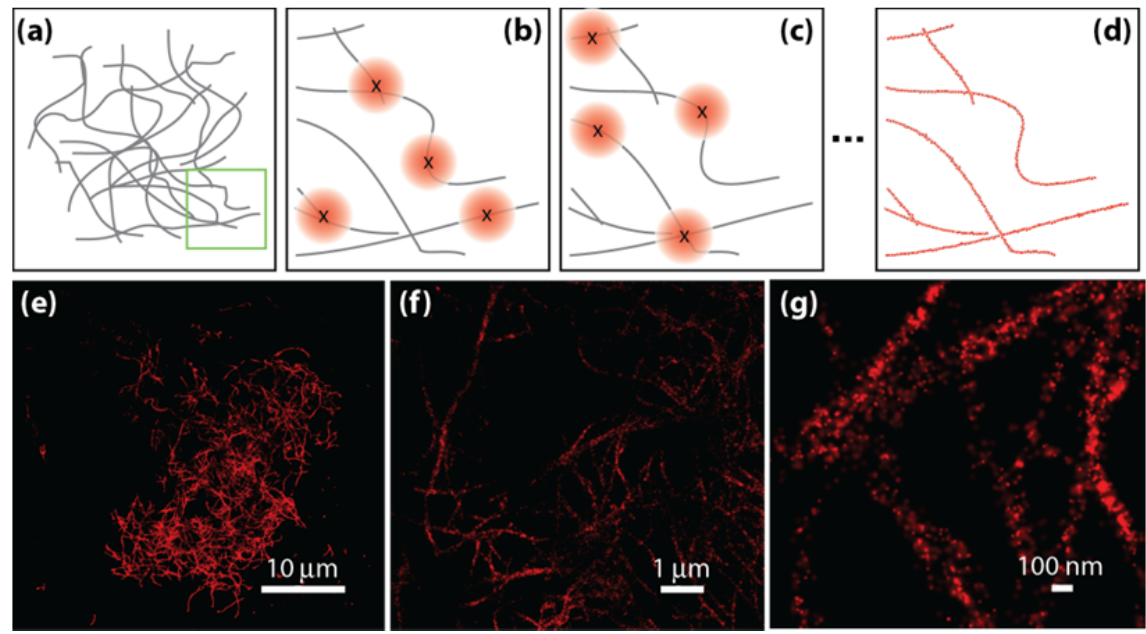

Figure 4. Principle behind photoswitching SRFM. A macromolecular structure (a) is labeled with fluorescent molecules, which are randomly switched between emissive and dark states, causing sparse collections of the fluorescent molecules to be imaged in each frame $(b-c)$. The molecules can be localized with high accuracy, and a super-resolution image $(\mathrm{d})$ is reconstructed using the locations of all molecules imaged. (e-g) Sample SRFM reconstruction images showing sub-diffraction sized fibrils. Adapted from Reference (101)

\section{Experimental implementations of advanced-microscopy techniques}

\subsection{Elucidation of cellulose structure and biochemical composition}

Cellulose, in the form of elementary microfibrils, constitutes the core structural component of plant cell walls. As such, the fundamental crystalline packing of glucan chains and microfibril structure determine many of the biological, chemical and physical characteristics of plant derived cellulosic materials. Despite the abundance of plant biomass and its importance to plant biology and the food, pulp and paper, textile, bioenergy and green materials industries, the structure of cellulose in plant cell walls is not known in detail.(102) This stems primarily from the reduced availability of techniques that can access the nanoscale structure of cellulose in vitro and in vivo under hydrated conditions, which preserve the natural arrangement of the glucan chains and their aggregated fibril forms. Over the past couple of decades, there has been increased interest in the use of advancedmicroscopy techniques to study the structure of cellulosic materials both in their natural or treated forms. In particular, AFM has been used extensively to gain insight into both the arrangement of microfibrils within the plant cell wall environment as well as into the crystalline packing of the individual glucan chains that compose these microfibrils.

As early as 1996 Kirby and collaborators used AFM to image the hydrated cell wall structure of apple, carrot, water chestnut and potato parenchyma.(103) In this seminal study, it was demonstrated for the first time that AFM could be used to effectively image and study the structural arrangement of cellulose within partially hydrated plant cell walls (Figure 5). 

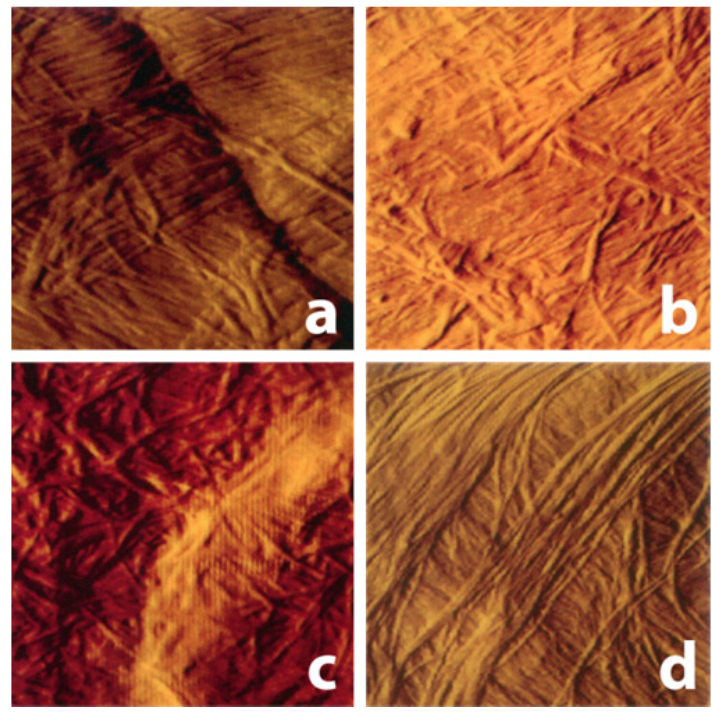

Figure 5. AFM error signal images of (a) apple, (b) carrot, (c) potato, and (d) water chestnut parenchyma cell walls show the polylaminate arrangement of cellulose microfibrils. All images are approximately $1 \times 1 \mu \mathrm{m}$. Reproduced form reference (103) with permission.

The topographical and error AFM images obtained from the cell wall of different plant species showed layers of aligned fibrous structures, which on the basis of size and shape were taken to be cellulose microfibrils. These microfibrils could be observed both in freshly prepared samples and in samples that had been stored frozen. The observation of the laminate arrangement of microfibrils with different orientations confirmed the previously held assumption that cell walls are polylaminate structures. It also marked the beginning of the use of AFM as a technique to study the structure of cellulosic materials. Just a year later Pesacreta et al. used tapping-mode AFM to investigate the structure of cotton fibre cell walls both in air and under water.(104) They reported widths for dry primary cell wall microfibrils in the 25-40 $\mathrm{nm}$ range, while they were able to observe striations within the cotton fibre with widths in the 5-7 nm range under completely hydrated conditions. These striations were considered to be the first observations of elementary cellulose fibrils. Later, Thimm et al. conducted AFM imaging of live celery parenchyma cell walls and studied the effect of dehydration on microfibril size.(105) Under complete hydration, microfibrils as small as 6-8 $\mathrm{nm}$ were observed. However, they observed that dehydration increased the observed microfibril size, and postulated an increase in fibre diameter through the coalescence of smaller fibrils and through the coating of the fibres with other polysaccharide components. A study of partially hydrated cell walls from the angiosperms Arabidopsis thaliana (thale cress) and Allium cepa (onion) by Davies and Harris also showed that intact microfibrils had widths of 5.8 and $4.4 \mathrm{~nm}$ respectively.(106) Furthermore, they showed that treatments to remove pectic polysaccharides reduced the size of the Arabidopsis microfibrils to $3.2 \mathrm{~nm}$. From these measurements they concluded that each of the observed intact microfibrils consisted of a single crystalline core or crystallite. Recent AFM experimentation 
by Ding et al. with corn parenchyma have given much higher resolution images of the cell wall structure.(107) In this study, in addition to elementary microfibrils with $3 \times 5 \mathrm{~nm}$ rectangular cross-sections, they identified cell wall depressions or "pits" with diameters in the micron and depths in hundreds of nanometer range, and macrofibrils that consist of bundles of smaller fibrils. From these observations, they have proposed a model for cellulose synthesis where in growing tissue elementary glucan chains synthesized from CesA protein rosettes aggregate to form macrofibrils, which at later stages disperse into the smaller bundles of elementary chains that constitute the core cellulose microfibrils coated by pectic polysaccharides. Furthermore, form their observations of the cross-sectional dimensions of cellulose microfibrils and computational models they proposed a model where each microfibril consists of 36 glucan chains that assemble through hydrogen bonding and van der Waals forces into the crystalline core and sub-crystalline shell of elementary microfibrils.(107) This model conflicts with recently obtained wide angle X-ray scattering and small angle neutron scattering data from spruce wood cell walls that suggests that elementary microfibrils consist of 24 glucan chains.(102) While there is still controversy about the overall dimensions of the structural components of the cell wall, it is without a doubt that AFM has yielded key insights and will continue to provide useful information of the arrangement of cellulose within plant cell walls. In particular, future implementations where AFM tips are functionalized to detect specific polysaccharides or proteins could provide a richer nanoscale visualization of the cell wall, its biochemical attributes and its biological function.

AFM imaging has also been used to study the fine structure of individual glucan chains and their crystalline arrangement within elementary microfibrils. These studies have been primarily performed with cellulose crystallites extracted from Valonia ventricosa, a green alga that is accepted as the standard source of cellulose I because its large crystallites consist of highly ordered 1200-1400 individual glucan chains.(108) The pioneering work of Hanley and collaborators used the Fourier transform of AFM images to show for the first time the periodicity corresponding to the unit cell of glucan chains in Valonia crystals.(109) Later on, Baker et al. imaged Valonia crystals in water and propanol and reported the direct visualization of the molecular periodicity within glucan chains and the intermolecular periodicity of their crystalline packing.(110) The imaged crystals appeared to be $5-12 \mathrm{~nm}$ high and 20-100 $\mathrm{nm}$ wide; the discrepancy of the latter dimension with previously reported sizes was attributed to tip-broadening effects. Upon close inspection, the surfaces of these crystals, which at low magnifications seemed smooth, showed corrugations that ran across the crystal. Three spacings were identified in the ultra-high resolution deflection AFM images and their corresponding Fourier Transform plots: the glucose interval with a periodicity of $0.52 \mathrm{~nm}$, the cellobiose repeat with a periodicity of $1.04 \mathrm{~nm}$ and the intermolecular spacing between glucan chains of $\sim 0.6 \mathrm{~nm}$. $(109,110)$ Furthermore, the triclinic arrangement of the glucan chains in the $\mathrm{I}_{\alpha}$ cellulose allomorph predominant in algal cells was observed as a $0.26 \mathrm{~nm}$ shift of the cellobiose repeat unit along the chain axis. These observations were further compared with computational models of the triclinic arrangement of the glucan chains and the measured periodicities were found to be in good agreement with the predicted spacings. $(111,112)$ While these studies together provided direct 


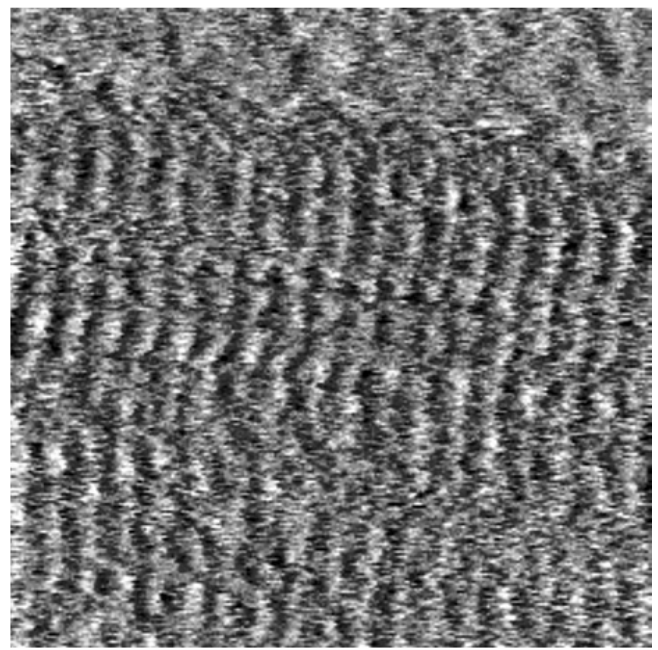

Figure 6. The AFM deflection mode image of a Valonia crystal surface shows spacings between the corrugations of $0.55 \mathrm{~nm}$, in good agreement with the spacing between glucose units obtained through crystallography. Glucan chains in the crystal run in the left to right direction. Reproduced from reference (110) with permission.

information of the intra and intermolecular spacing of glucan chains in cellulose $\mathrm{I} \alpha$ crystals from an algal source, no work to date has been performed to observe the spacings in cellulose derived from tunicates or plants, which have cellulose crystallites composed predominantly of the cellulose $\mathrm{I}_{\beta}$ allomorph. Furthermore, higher resolution probes and more sensitive instruments should allow the acquisition of even higher resolution images, which might help identify the regions of plant cell wall material containing coexisting phases of both allomorphs.

Another area of research that has benefited from advanced-microscopy techniques has been the investigation of how the cellulose structure changes as a result of different mechanical or chemical treatments. This is particularly important for the characterization of pulping processes, the pretreatment of plant biomass, and for the development of novel green materials with enhanced physical and chemical properties based on nanocrystalline cellulose. Pulping is the mechanical or chemical process used to separate cellulose from plant biomass and its outcome, a lignocellulosic fibrous material called pulp, is the primary raw material for papermaking. A number of studies have utilized AFM to investigate the effect of chemical and mechanical treatments used in the pulping process on the resulting cellulose fibres. Koljonen and collaborators used AFM to study the structure of spruce that had been mechanically treated to produce unbleached pressure groundwood pulp (PGWP), thermomechanical pulp (TMP) and chemothermomechanical pulp (CTMP).(113) They found that the surfaces of all pulps exhibited heterogeneous morphology, with the surfaces of PWGP and TMP showing fibrilar and granular structures, while CTMP showed a surface almost completely covered by granular material. This granular structure was interpreted as lignified material that could be removed after peroxide bleaching. Similarly, Gustafsson and 
collaborators studied the effect of cooking and bleaching on Kraft pulp produced from spruce biomass.(114) It was observed that spruce pulp that had been cooked for short times (15-30 minutes) exhibited highly granular surfaces with granules, containing lignin and extractives, of 40-300 $\mathrm{nm}$ size. Increasing the cooking time reduced the amount and size of the granules, but did not eliminate them completely. Further acetone extraction and delignification steps effectively removed the granular material, exposing the underlying fibrilar cell wall construction corresponding to structural polysaccharides. The irregularity observed in fibril size was attributed to the presence of both crystalline cellulose and amorphous hemicellulose.

More recent studies have shown that image analysis techniques can be used to extract more quantitative information from AFM images taken from cellulose samples that have undergone different treatments. Fahlen and Salmen have shown that image analysis techniques can evidence the change in size of cellulose aggregates in spruce wood pulp produced through kraft cooking in presence of different amounts of alkali.(115) Schmied and collaborators recently presented the use of a watershed algorithm to distinguish between cellulose fibre and precipitated lignin aggregates in AFM images taken from softwood Kraft pulps.(116) Through this analysis they were able to investigate the kinetics of lignin precipitation and the structure of these aggregates during the processes of pulping and bleaching. In addition to pulping, the effects of other processing techniques on cellulose structure have been given much attention recently, especially in the context of the pretreatment of biomass for the production of biofuels and bioproducts. For example, Eronen and collaborators characterized the changes induced in pure cellulose samples by the process of mercerization, where alkali induces a change in the crystalline cellulose packing and transforms cellulose I into the cellulose II allomorph.(117) They observed that fibrils imaged in mercerized samples appeared swollen and granular, but that the overall surface roughness of the cellulose samples decreased. These observations were correlated to confocal Raman imaging. AFM has also been recently combined with electron microscopy, $\mathrm{x}$-ray photoelectron spectroscopy and attenuated total reflectance Fourier transform infrared spectroscopy to study the effects of hydrothermal pretreatment on wheat straw biomass (118) and the effects of active oxygen and solid alkali pretreatment on corn stock biomass.

Similar AFM characterization has been used to determine the physical characteristics of cellulose nanocrystals produced through acid hydrolysis from different plant materials. For example, it has been shown that never-dry materials or materials that are dried under high relative humidity produce longer nanocrystals than those produced from dried materials, an effect ascribed to the tension induced by the drying process and the underlying supramolecular changes in the crystalline cellulose packing.(120) AFM characterization has also helped demonstrate that cellulose II nanocrystals can be produced through the dissolution of cellulose I in sulphuric acid followed by recrystallization under conditions where the acid concentration and treatment duration are carefully controlled.(121) The intense focus on the use of nanocrystalline cellulose for the production of green materials and materials with improved physical and chemical characteristics has spurred an increase 
in the research devoted to the nanoscale characteristics and structure of these crystalline structures. It is anticipated that advanced-microscopy techniques, such as AFM will play a fundamental role in understanding the nanoscale structure properties of these materials and in helping guide the development of new biocomposites.

\subsection{Elucidation of cell wall structure through protein adsorption}

Advanced microscopy techniques have been used to image the adsorption of proteins to cellulosic substrates, both with the intent of characterizing the inherent cell wall structure as well as studying the effects that different physical, chemical and biological pretreatments have on cellulosic substrates. In particular, fluorescence microscopy techniques have been widely used for these studies because they can selectively image fluorescently tagged molecules (e.g. antibodies, cell wall binding proteins) with very high sensitivity over the background presented by the cellulosic material. Furthermore, the availability of fluorescence techniques that can perform optical sectioning has enabled the precise localization of the fluorescent molecules in space and the reconstruction of threedimensional profiles of complex structures contained within cellulosic samples. The application of fluorescence techniques to image protein adsorption has even been extended down to the level of individual molecules, where specific molecular orientations can report on the crystalline arrangement of cellulose fibrils, and with the advent of super-resolution fluorescence microscopy it is foreseen that more detailed structural information from cellulosic substrates will be obtained through fluorescence with resolutions comparable to those achieved by electron microscopy.

Early microscopy studies of the structure of polysaccharide components within cell walls were conducted with whole xyloglucan $(X G)$ binding proteins, galactan-binding proteins or antibodies specifically raised against these cell wall components. One of the first studies of this kind, published in 1984 by Hayashi and Maclachlan, utilized a fluorescently labeled fucose-binding lectin to visualize the spatial distribution of $X G$ in a cellulose/XG macromolecular complex extracted from the elongating regions of etiolated pea.(122) The images obtained through fluorescence microscopy were compared to those obtained through radioisotope imaging and electron microscopy and it was determined that XG was present both on and between the cellulose fibrils of the complex. More recently, Brunecky and collaborators used the immunostaining technique with an XG-directed primary antibody and a fluorescent secondary antibody to study the changes in the distribution of xylan caused by dilute acid pretreatment through confocal laser scanning microscopy.(123) Using this technique they observed that the XG distribution, which was homogeneous in untreated cell walls, was drastically reduced in the center of the cell walls and increased in the middle lamella and the lumen of the cell walls of pretreated materials. Furthermore they observed a decrease in the overall fluorescence signal, which was consistent with the decrease in XG content of the bulk sample. A similar immunostaining and imaging approach has been reported for the visualization of the distribution of XG and galactan in developing (124) and mature tension wood poplar cells.(125) In these reports, the images obtained through fluorescence imaging were also compared to electron microscopy 
immunostained samples to gain higher resolution of the localization of these hemicellulose components within the cell walls. Despite the ability to visualize the distribution of hemicellulosic components within the cell wall at the micron level, the use of whole proteins and sequential staining with antibodies precludes the study of cell wall structure through fluorescence with high resolution. Because large proteins, such as antibodies, cannot efficiently penetrate interstitial spaces and pores with nanometer dimensions within the cell wall structure, smaller probes are needed to achieve better labeling and higher resolution of hemicellulosic components.

The search for smaller, more specific probes to image and characterize the structural arrangement of polysaccharides in plant cell walls has lead researchers to explore the use of glycoside hydrolase fragments as highly specific molecular imaging probes. Glycoside hydrolases, plant cell wall degrading enzymes produced by a range of bacteria and fungi, have complex molecular architectures consisting of one or more catalytic domains (CDs) and one or more carbohydrate binding modules (CBMs).(126) It is thought that the primary function of CBMs is to promote the attachment of the parent enzyme to its substrate, thus increasing the local concentration of the enzyme and enhancing its catalytic activity. CBMs have evolved in nature to bind to different polysaccharides encountered in the cell wall, as well as starches and other polysaccharides such as chitin that are encountered in a wide range of biological systems. Currently there are 64 families (or types) of CBMs catalogued as functional or putative carbohydrate binding motifs based on their amino acid and structural similarity, as identified in the carbohydrate active enzyme database (CAZy; http://afmb.cnrs-mrs.fr/cazy/).(127) The compact structure of CBMs and their specificity towards the targeted substrate of their parent enzyme have made CBMs prime candidates for the study of the structural arrangement of polysaccharides within the cell wall.

The first studies using CBMs as molecular probes for visualization utilized fluorescent antibodies raised against the CBMs or against motifs appended to them through protein engineering. Linder et al. utilized a recombinant construct where two cellulose-binding modules from Trichoderma reseii were linked together to bind specifically to cellulose and established a quick protocol to differentiate between Acanthamoeba, which produces cellulose, and other protozoan parasites that only produce chitin.(128) They visualized the bound CBMs through epifluorescence using an antibody sandwich assay consisting of a primary mouse anti-cellulase antibody and a secondary fluorescent anti-mouse antibody. In 2004, McCartney and collaborators conducted a study that systematically characterized the binding specificity of CBMs from families 2a (bind primarily to crystalline cellulose), 6 (bind to xylan), and 29 (interact with $\beta$-(1-4)-linked glucans, mannan, and glucomannan).(129) Using an antibody sandwich assay they were able to visualize the binding of CBMs within thin sections of maize cell walls through epifluorescence microscopy. In this study, they showed for the first time the ability to use recombinant his-tagged CBMs as flexible molecular probes to localize their polysaccharide targets and study the complex cell wall structure in plants. A couple of years later, the same group interrogated the binding specificity of six xylan-binding CBMs (from families 2b, 4, 6, 15, 22 and 35) for their target polysaccharides within the cell walls of a variety of species, such as wheat, flax, tobacco, 


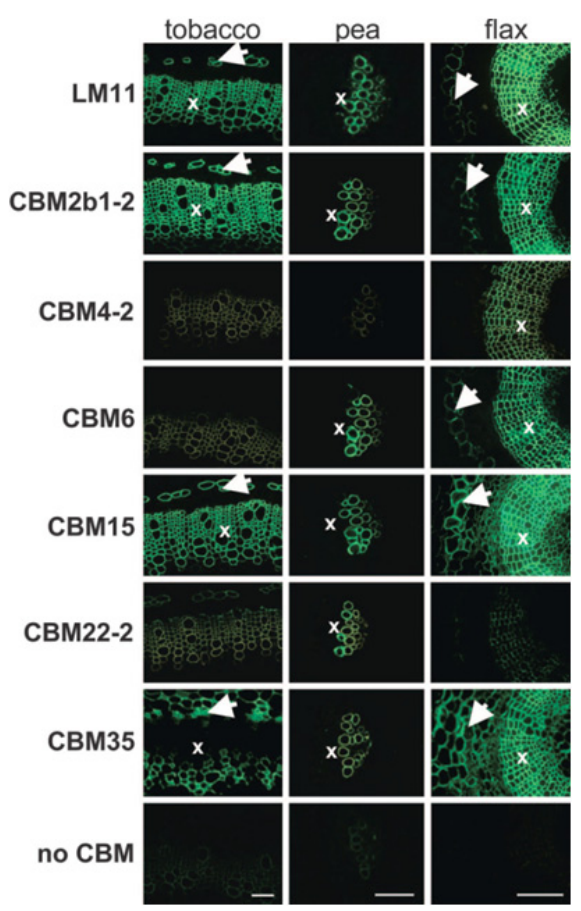

Figure 7. Epifluorescence microscopy of CBMs and monoclonal antibody LM11 binding to secondary cell walls of vascular tissues in thin sections of tobacco, pea, and flax stems. Scale bar $100 \mu \mathrm{m}$. Reproduced from reference (130) with permission.

pea, and maize through epifluorescence microscopy (Figure 7).(130) In this study they found that the CBMs tested showed significant differences in the recognition of plant material, and stained the xyloglucans in the primary and secondary cell walls to different extent. They postulated that the context in which the xyloglucans are encountered in the plant is a determinant factor for the ability of CBMs to recognize their targets, and that the variability in recognition in plants must be related to the ability of the different enzymes to hydrolyze xyloglucans in different species. Recently, similar immunofluorescence labeling of cellulose directed CBMs and epifluorescence imaging approaches have been used to study the structural arrangement of the crystalline cellulose contained within the G-layer of tension wood derived from poplar and birch (131) and to study the changes in crystallinity of cellulose II in lyocell fibers derived from eucalyptus wood pulp as they were subjected to $\mathrm{NaOH}$ treatment.(132) Altogether these studies have shown that CBMs are suitable probes to study the structure of cellulosic materials derived from plant cell walls through fluorescence microscopy.

Over the past decade, a number of different labeling approaches have been pursued to directly tag CBMs with fluorescent moieties in order to avoid the need for multiple antibody incubation steps. Daniel and collaborators first reported the direct labeling of T. reseii Cel7A CBMs with organic dyes (FITC/TRITC) for the visualization of crystalline cellulose within the 
G-layer of tension wood.(131) Similarly, Filonova and collaborators directly tagged synthetic xylan-binding CBMs to map the carbohydrate distribution within wood (birch and pine) sections and Kraft pulp fibers (birch).(133) The use of CBMs that were directly labeled with fluorescent molecules effectively reduced the number of sample preparation steps and allowed the acquisition of higher resolution images through epifluorescence microscopy. Yet, a concern of direct labeling of these molecular probes with organic dyes is that because of the randomness of the tagging process, reduced binding could result from labeling of residues that are involved in binding. A second approach that has been tested to directly label CBMs has been tagging them with quantum dots (QDs), nanometer-sized colloidal semiconductor particles with tunable fluorescent emission properties, which are directed towards specific amino acid sequences appended to the CBM. In 2006 Zhang reported the first images of QDCBM assemblies bound to crystalline cellulose and showed that these constructs could be used in combination with near field scanning optical microscopy to obtain fluorescence images with enhanced spatial resolution.(134) More recently, $\mathrm{Xu}$ et al. have reported the labeling of two CBMs from families 2 and 3a with a range of QDs with different spectral properties and their imaging using epifluorescence microscopy.(135) This study used the labeled CBMs to visualize Valonia cellulose crystals with diameters of $20 \mathrm{~nm}$ and lengths in the 15 to $20 \mu \mathrm{m}$ range, and showed that the QD-CBM assemblies preferentially bound to the planar face of the cellulose crystal in the form of linear arrays. Furthermore, $\mathrm{Xu}$ and collaborators observed that by using different QD sizes they could control the spacing between CBMs adsorbed on the cellulose surface, which indicated that the steric hindrance between neighboring QDs was the limiting factor for the density of bound CBMs. They also suggested that due to the bright emission of these QD-CBM constructs, they could find use in SM studies to interrogate the catalytic and non-catalytic motion of CBMs on cellulose surfaces.

In 2006, Ding et al. further introduced the use of CBM-fluorescent protein (FP) fusion constructs as molecular probes for the characterization of the complex structure of cell walls and other biomaterials.(136) In this pioneering study, CBMs from families 3, 6, and 20 labeled with QDs or expressed as constructs that incorporated the fusion of either a green or red fluorescent protein (GFP or RFP) were used to label native and phosphoric acid treated Valonia cellulose crystals, starch granules, cellulose/tamarind xyloglucan composites, and maize primary cell walls (Figure 8). FP-CBM constructs were found to be highly specific and bound to their intended targets on complex biomaterials containing mixtures of cellulose, starch and xyloglucan. Since the publication of this work, labeling of CBMs with FPs has become a popular solution for the direct tagging of cell wall binding fragments for a wide variety of studies. CBM-FPs have been used to study the effect of different pretreatments on the structure of plant cell walls. For example, in a report by Porter et al. cellulose and starch directed CBM-GFP/RFP fusion proteins were used to visualize the effect of the wet milling process on corn fibers through confocal microscopy.(137) They observed that the binding of the starch directed CBM-FP correlated well with the remaining starch content in the treated corn fibers and that the cellulose directed CBM-FP construct could not bind significantly to any of the wet milled materials, but would bind well to samples where the starch and most of the hemicellulose had been removed. This evidenced the need to remove as much of the starch and hemicellulosic materials as possible if cellulolytic enzymes are to efficiently 


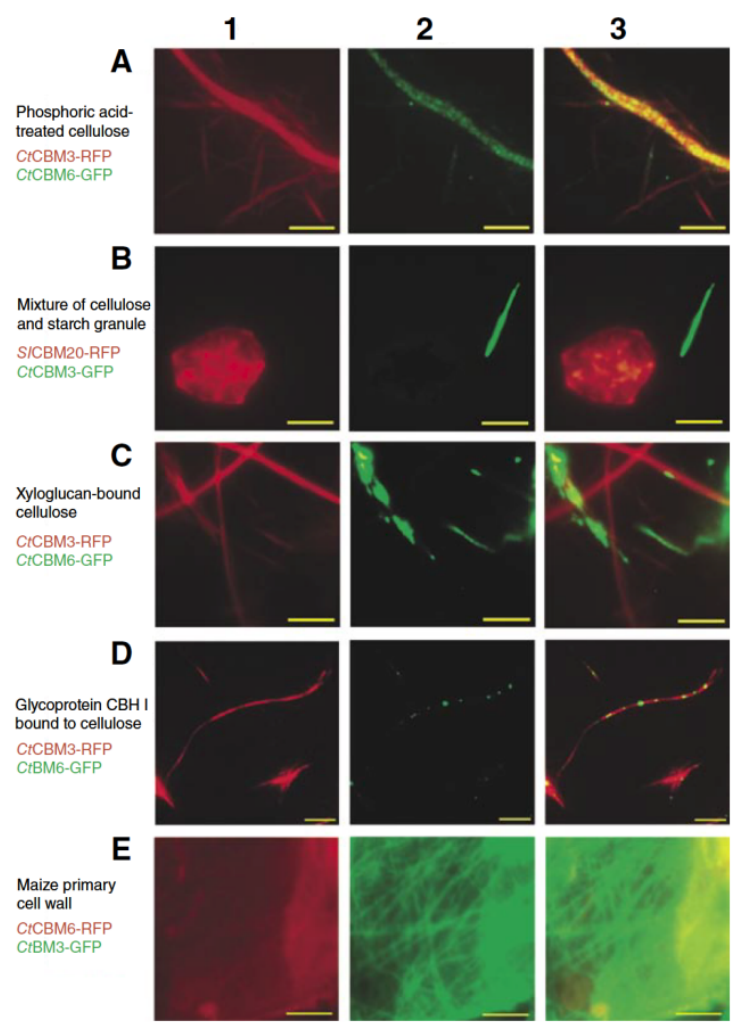

Figure 8. Fluorescence images showing the recognition and binding of two different CBMs to various materials. Column 1 shows fluorescence from RFP labeled samples, column 2 shows the fluorescence from GFP labeled samples, and column 3 shows an overlay of both channels. Each row of images depicts a different material incubated with two distinct CBMs from families 3 (binds to crystalline cellulose), 6 (bind to amorphous cellulose and xyloglucan), and 20 (bind to starch). All scale bars represent 5 microns. Reproduced from reference (137) with permission.

depolymerize crystalline cellulose fibrils within the plant cell wall. Kawakubo et al. also used constructs of family 3 and 28 CBMs fused to cyan fluorescent protein (CFP) as markers for the visualization of crystalline and amorphous cellulose on the complex surfaces of wood tissues pretreated with $\mathrm{NaOH}, \mathrm{NaOH}-\mathrm{Na} 2 \mathrm{~S}$ (kraft pulping), hydrothermolysis, ballmilling, and organosolvolysis.(138) Their assay with CBM-CFPs was proposed as a facile way to measure the extent of exposure of fibrous structures, which are the putative initiation sites of hydrolysis and saccharification in chemically delignified wood pulps. Other applications of CBM-GFP constructs in recent years have been in the study of the distribution of crystalline cellulose in the cell walls within tracheary elements of Zinnia elegans,(139) and in the visualization of cell wall regeneration in algal protoplasts.(140)

Up to date, most of the studies conducted to elucidate the structural characteristics of plant cell walls through CBMs that specifically bind to structural polysaccharides have been performed 
using either epifluorescence or confocal microscopy. Yet, the development of new approaches to perform targeted labeling of CBMs has opened the door for the use of additional forms of advanced fluorescence microscopy techniques. This has already been explored by Dagel and collaborators, who have used a single molecule fluorescence technique termed Defocused Orientation and Position Imaging (DOPI) to visualize the orientation of single CBM-GFP constructs as they bind to Valonia crystals.(141) In this study they used CBMs from families 1, 2 and 3, and showed that binding of these modules was specific for the hydrophobic facet of the cellulose crystals and that they display a preferred orientation with respect to the fiber axis.

Another advanced-microscopy technique that holds promise for the study of plant cell wall structure with molecular resolution is Super Resolution Fluorescence Microscopy (SRFM). In particular, the reconstruction of molecular resolution maps of cellulosic materials is possible through the direct labeling of the targeted polysaccharides (Figure 9) or through binding of molecular reporters such as highly specific CBMs and whole proteins (Figure 9).(101,142) Advanced fluorescence microscopy techniques like these are sure to play an important role in the molecular characterization of the structure and composition not only plant materials, but also of biocomposites and biomaterials.
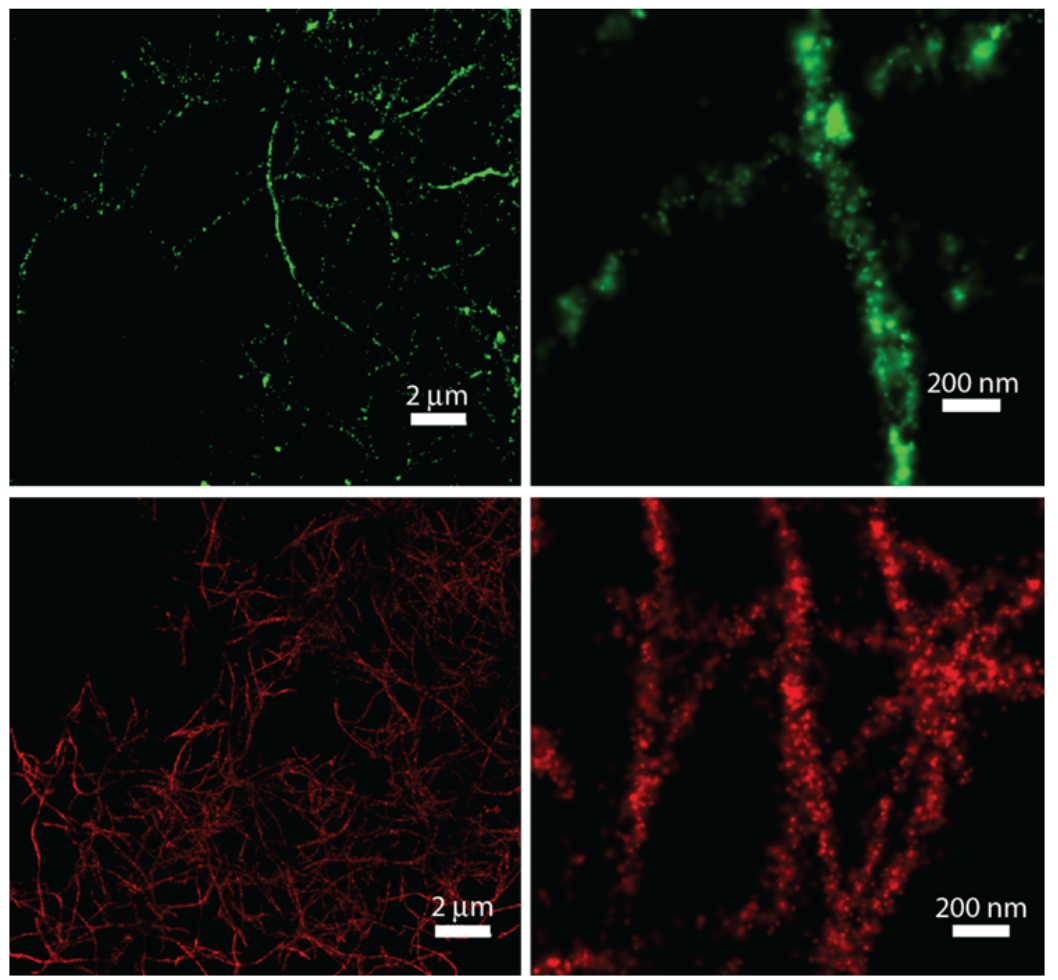

Figure 9. Super-Resolution Fluorescence Microscopy images of cellulose microfibrils (top, direct imaging of cellulose through DTAF labels) and bound cellulases (bottom, T. fusca Cel9A) reveal the nanoscale structure of crystalline cellulose. Adapted from Reference (101). 


\subsection{CBM and cellulase binding kinetics}

The use of highly sensitive cameras in conjunction with stable illumination sources and efficient fluorescent emitters has enabled the quantitative assessment of fluorescence intensity measurements. This has been used to acquire time resolved fluorescence microscopy images for the study of CBM and cellulase binding kinetics. Pinto et al. conducted the first studies using time-lapsed epifluorescence microscopy to study CBM binding kinetics in 2006.(143) In this study they demonstrated a method for the gram-scale production of CBMs derived through mild proteolysis from $T$. reseii $\mathrm{CBHI}$ cellulases contained within a commercial cellulolytic cocktail. The purified CBMs were subsequently labeled with an organic dye (FITC) and were used to study CBM binding kinetics on a variety of cellulosic substrates. Image analysis of the fluorescence intensity of the samples showed that CBMs bound preferentially to amorphous cellulose, that the CBMs displayed saturation adsorption kinetics, and that CBM binding was reversible, but that the rate of desorption was slow. The same group a year later published a fluorescence microscopy image analysis method to quantitatively determine the concentration of adsorbed CBMs on cellulosic surfaces.(144) In this method, the fluorescence intensity of images obtained from solutions with known concentrations of fluorophore, which were confined in a chamber with known volume, were used as calibration to extract the concentration of fluorescent CBMs bound to thin cellulose films. Their image analysis routines allowed the quantification of CBM adsorption to homogeneous cellulose surfaces and the reconstruction of binding isotherms that matched those obtained from bulk measurements. This methodology has recently been extended to the quantitation of CBM binding onto cellulosic fibers through epifluorescence and laser scanning confocal fluorescence microscopy.(145) These studies have demonstrated the ability to use fluorescence microscopy and time-lapsed image acquisition to measure the binding kinetics of cellulose binding modules.

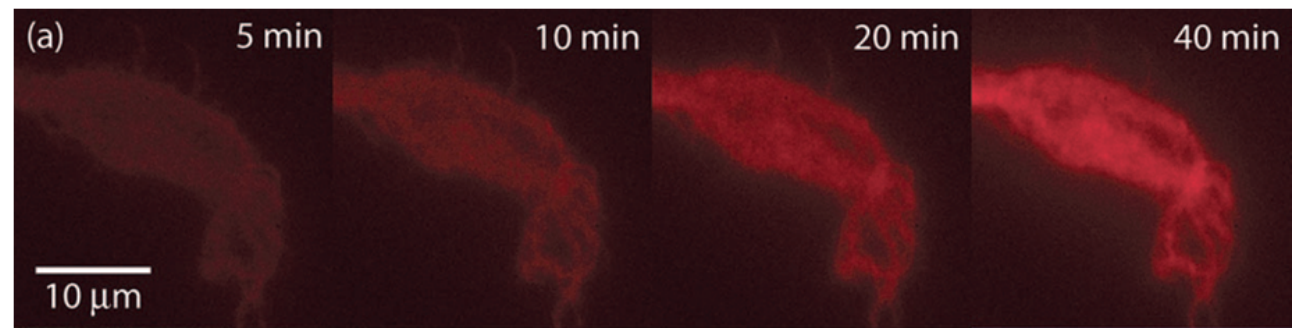

Figure 10. Epifluorescence imaging of real time binding of Cel6B onto immobilized cellulose microfibrils. Representative images of points in time-lapsed experiments in red channel fluorescence show AF-647 cellulase binding onto surface immobilized cellulose. Reproduced from reference (146) with permission.

Quantitative fluorescence microscopy has also been demonstrated for the study of binding kinetics of whole cellulases. In 2008, Moran-Mirabal et al. reported the fluorescent labeling of Thermobifida fusca Cel5A (classical endoglucanase), Cel6B (exoglucanase), and Cel9A (processive endoglucanase) cellulases and their use to study binding kinetics on 
fluorescently labeled bacterial microcrystalline cellulose (BMCC) that had been immobilized on a micropatterned surface.(146) The images obtained through epifluorescence microscopy showed uniform binding of all three cellulases on the surface of the cellulose fibrils, which pointed to binding mediated primarily by the CBM. The time lapse image sequences also evidenced that binding kinetics obtained from individual cellulose fibrils, where cellulases can bind readily, closely followed a saturation binding model, while binding kinetics obtained from cellulose mats and particles, where the porous structure hinders cellulase accessibility to internal cellulose surfaces, significantly deviated form it. This pointed to a strong influence of the physical structure of cellulosic substrates on the ability of cellulases to bind to and ultimately hydrolyze cellulose. A similar approach was taken by Zhu et al. to study the binding kinetics of T. fusca cellulases on thermally pretreated wood particles.(147) Confocal laser scanning fluorescence microscopy was used in this study to capture threedimensional images of the autofluorescence arising from the lignocellulosic particles and fluorescence from cellulase binding onto the cellulose. Fluorescence images were recorded in three spectrally different channels and a deconvolution algorithm was introduced to separate the autofluorescence from the bound cellulase signal. In this way it was observed that cellulases bind preferentially to certain areas of the pretreated particle, arguably those containing easily accessible amorphous cellulose. The time-lapse fluorescence intensity data extracted through image analysis provided a quantitative measure of bound enzymes over time, and were fitted to a transient enzyme-binding model. However, the transient binding model could only be fitted to the initial binding phase and the authors concluded that a more complete model was needed to further explain the binding patterns observed in the complex structure of pretreated lignocellulosic particles.

In recent years advanced imaging techniques have started to be used to study cellulasecellulose interactions with higher spatial and temporal resolution. The group of Ragauskas has pioneered the use of Förster Resonance Energy Transfer (FRET) for the study of cellulase-cellulose interactions(148) and cellulase-cellulase colocalization.(149) Their first study demonstrated the ability to investigate the temperature dependence of cellulase binding to cellulose by monitoring the fluorescence emission change of donor (or acceptor) as the bound cellulase came into close proximity of the fluorescent labels grafted onto the cellulose substrate.(148) This study demonstrated the use of FRET in a homogeneous liquid environment, with carboxymethyl cellulose as a soluble substrate. In a subsequent study, Wang et al. demonstrated the ability to use FRET together with acceptor photobleaching and spectral unmixing to determine the extent of colocalization of cellulase on cellulose fibrils. In this study, cellulase labeled with two spectrally distinct dyes was incubated with cellulose fibrils and epifluorescence images were acquired before and after bleaching of the donor dye.(149) Although this study was intended as a proof of concept, the authors demonstrated that by changing the concentration of the donor dye on the cellulose fibrils they could observe changes in the acceptor fluorescence, indicating that the cellulases labeled with the different dyes were localized next to each other within a radius corresponding to the molecular size of the enzyme. Another advanced imaging technique that has been successfully applied to study the binding kinetics of cellulases is fluorescence recovery after photobleaching (FRAP). Moran-Mirabal et al. pioneered the use of FRAP with a confocal 
laser scanning microscopy setup to study the binding reversibility of $T$. fusca cellulases to fibrils and mats composed of bacterial microcrystalline cellulose.(150) This study not only demonstrated that cellulase binding to cellulose is reversible, but also showed that a certain fraction of all adsorbed cellulases is irreversibly bound to the surface. Furthermore, it was the first implementation of fluorescence microscopy imaging of cellulases at temperatures where significant catalysis occurs. The effect of temperature was significant in the binding kinetics observed, especially for processive enzymes, where higher temperatures increased the rate of desorption of the reversibly bound enzymes, while at the same time increasing the irreversibly bound fraction. The implementation of advanced microscopy techniques for the study of binding kinetics is still in its initial stages, and we can foresee an increase in the use of non-traditional techniques to gain a deeper understanding into the mechanisms at play in cellulase-cellulose interactions.

\subsection{The molecular transport in cellulosic substrates}

The study of diffusion and transport processes on cellulosic substrates is arguably the area that has benefited the most from the employment of advanced microscopy techniques. Scanning probe microscopy as well as fluorescence techniques have been used since the end of the 90 's to study the molecular diffusion of glycoside hydrolases on cellulosic surfaces, the processive motion of cellulases as they catalyze the breakage of glycosidic bonds, and the transport of biomolecules through the porous structure of plant derived materials. Jervis and collaborators conducted the first study of the diffusion behaviour of bacterial cellulases and their isolated CBMs on microcrystalline cellulose using FRAP.(151) In this study, they bound fluorescently labeled cellulases and CBMs to a cellulose mat and washed the excess unbound cellulases before bleaching. Areas of the cellulose mats were bleached with a high intensity laser pulse and the recovery of fluorescence was monitored over time. Because it was assumed that the cellulases were irreversibly bound to the cellulose, all recovery was attributed to diffusion along the surface of the cellulose fibrils. Jervis et al. reported a cellulase mobile fraction of $70 \%$, and diffusion coefficients ranging from $2 \times 10^{-11}$ to $1.2 \times 10^{-10}$ $\mathrm{cm}^{2} / \mathrm{s}$ for cellulases and isolated CBMs. They further concluded that the surface diffusion of cellulases was not a rate limiting process for the hydrolysis of cellulose. A similar FRAP experimental approach was used by Cuyvers et al. to probe the surface diffusion of different Bacillus subtilis xylanase mutants on wheat and oat spelt xylans.(152) They observed that the wild-type enzyme was fully mobile and showed complete recovery in bleached areas, whereas an inactive mutant displayed binding but no recovery over the xylan surface. Thus, they concluded that catalytic activity was essential for the mobility of the enzyme on the xylan surface. They also observed that higher binding affinity resulted in slower or hindered recovery. Recently, Moran-Mirabal et al. have used FRAP and single molecule tracking (SMT) techniques to study the surface diffusion of $T$. fusca exo- and endocellulases on bacterial micro-crystalline cellulose at a range of different temperatures.(153) In this study, they used carefully controlled environmental conditions to conduct FRAP experiments and found that $T$. fusca cellulases exhibit limited surface diffusion even at temperatures where significant catalysis occurs. It was shown that when the unbinding and rebinding contribution to fluorescence recovery is removed, a significant fraction of cellulases is 
immobile and recovery is significantly diminished. This suggested that surface diffusion is limited for T. fusca cellulases. In order to confirm these observations, SMT experiments were conducted where the motion of individual enzymes was tracked over time lengths in the order of minutes. They observed that most of the cellulases remained immobile, and that those that exhibited significant mobility displayed heterogeneous behaviours (Figure 11). The authors suggested that these could be explained by the differentiation of the observed displacements into hopping and sliding motions. This study for the first time showed that the dynamics of cellulase motion on cellulose surface are highly heterogeneous and cannot be explained by surface diffusion alone. Furthermore, this study showed that long-range non-catalytic displacements could be tracked using single molecule fluorescence techniques.
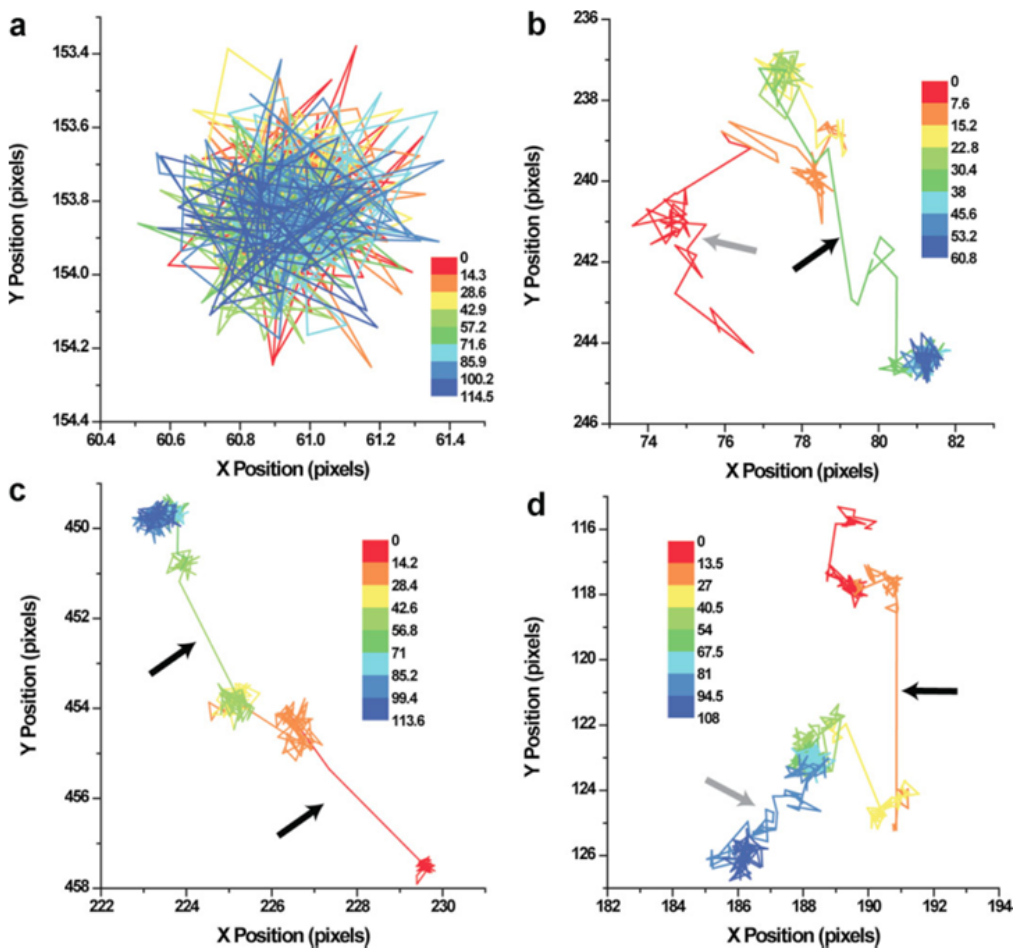

Figure 11. Single molecule tracks show that $T$. fusca cellulases exhibit complex motion patterns that can be related to molecular states. Sample tracks for: (a) immobile Cel5A cellulase, (b) mobile Cel5A cellulase, (c) mobile Cel6B cellulase, and (d) mobile Cel9A cellulase. All tracks displayed were recorded at $45^{\circ} \mathrm{C}$. Color of tracks represents time in seconds $(0.4 \mathrm{~s} /$ frame $)$. Black arrows highlight displacements that correspond to the putative hopping motion, while gray arrow highlight displacements consistent with the putative sliding motion. Reproduced from reference (153) with permission.

The development of High-Speed-AFM (HS-AFM) over the last decade has recently enabled the direct visualization of the displacements resulting from the processive catalytic action of T. reseii Cel7A cellobiohydrolase on crystalline cellulose fibrils. These studies, pioneered by Igarashi et al. have already given very valuable insight into the processive action of 
cellulases as they hydrolyse glycosidic bonds. In a seminal study published in 2009, Igarashi et al. for the first time visualized the processive displacement of individual Cel7A molecules on Cladophora cellulose crystals and reported an average processive speed of $3.5 \mathrm{~nm} / \mathrm{s}$ and maximum displacements for a single enzyme on the order of $50 \mathrm{~nm}$.(27) They further demonstrated that the same sliding motion could be observed for isolated catalytic domains, but not for inactive mutants. This confirmed that the observed motion arose solely from catalysis and not from non-catalytic surface diffusion. In addition, they observed that the mutation of a tryptophan residue at the entrance of the catalytic cleft significantly reduced the enzyme's mobility, which demonstrated that this residue that promotes the loading of the cellulose fibril into the cellulase catalytic cleft is also key component to the processive motion of cellulases. In a subsequent study, Igarashi et al. presented results of T. reseii Cel7A processivity measurements obtained using an improved version of the HS-AFM apparatus.(28) The enhanced temporal and spatial resolution afforded by the improved HSAFM allowed them to visualize two different Cel7A populations, one that represented enzymes exhibiting processive motions with an average speed of $7.1 \mathrm{~nm} / \mathrm{s}$ and another that represented enzymes halted on the cellulose surface (with speeds of $-0.3 \mathrm{~nm} / \mathrm{s}$ ). They also observed that mobile enzymes exhibited stop-and-go motions that sometimes led to the accumulation of halted enzymes in single file ("traffic jams") on the cellulose fibril surface. The observed traffic jams could be overcome if a significant number of enzymes accumulated, after which the enzymes could proceed with their processive motion. This study further investigated the effect of the cellulose allomorph type on cellulase motion and found that cellulose III allomorphs were able to accommodate higher density and more fluid processive motion of the cellulases. Finally, they investigated the effect of the co-incubation of Cel7A cellulases with Cel6A exocellulase, and found that there was a synergistic effect where the addition of Cel6A reduced the possibility of "traffic jams" and opened up more molecular cellulose lanes for Cel7A cellulases to diffuse in. Together these two studies have shown for the first time that it is possible to visualize the processive motion of cellulases, and have opened the door for subsequent studies of the molecular motion of other glycoside hydrolases.

The visualization of the diffusion of biomolecules through cellulosic substrates can provide significant insight into the transport limitations that enzymes encounter during the process of biomass saccharification. Yet, there are few examples of studies that use advanced imaging techniques to probe the diffusion of biomolecules and polymers into the porous structure of cellulosic materials. This is partly due to the fact that imaging the three dimensional structure of complex lignocellulosic materials through optical techniques is challenging. On one hand, the presence of lignin introduces large background signals in visible channels arising from autofluorescence. On the other, optical sectioning techniques can only probe a small fraction of thick samples because these substrates tend to have high refractive indices and can scatter much of the incident light and the emitted fluorescence. Despite these difficulties, Horvath et al. have recently reported a comprehensive study of the diffusion of cationic polymers into anionic cellulosic fibers using confocal laser scanning microscopy.(154) Through the use of polymer chains with different lengths and different charge densities they were able to show that the charge density of the polyelectrolyte had a larger effect on the diffusion times than did 
the molecule length. They also observed that the use of high ionic strength solutions could completely screen the interaction between the polyelectrolytes and the cellulosic fibers, leading to faster penetration into the porous structure of the substrates. This study demonstrated the ability to visualize diffusion processes occurring within the complex porous structure of cellulose, and has set the stage for future studies on the diffusive behaviour of glycoside hydrolases during the process of saccharification. It is anticipated that a number of novel advanced microscopy techniques, such as second harmonic generation and multiphoton fluorescence microscopy will find applications in the study of diffusion and transport processes with real time capabilities within cellulosic materials.

\subsection{Cellulose depolymerization by glycoside hydrolases}

The structural polysaccharides present in plant biomass are an attractive source of fermentable sugars that can be employed in the production of biofuels, chemicals and bioproducts. Yet, it has been recognized that the greatest bottleneck in conversion technology for the release of soluble sugars is the inherent recalcitrance to decomposition of the cellulose within the plant cell wall.(155) Two specific aspects that contribute to the recalcitrance of lignocellulose are: (i) the tight bonding between glucan chains in the highly crystalline cellulose microfibrils and (ii) the structural arrangement of cell wall components, which gives rise to pores and crevices with dimensions from micrometers to nanometers that can hinder the transport of catalysts from solution to the insoluble cellulose. Thus, understanding the mechanisms by which hydrolytic and non-hydrolytic proteins disrupt the structure of recalcitrant cellulosic substrates can be an important step towards designing more efficient and economical biomass conversion strategies. This has resulted in an increased interest in methods for visualizing the process of cellulose depolymerization in real time and with high spatial resolution. Such techniques could yield invaluable information to help elucidate some of the molecular mechanistic details of biomass processing. In recent years, a number of studies have used advanced microscopy techniques to study the depolymerization of cellulose by glycoside hydrolases and obtain qualitative and quantitative information about the catalytic processes occurring at the interface between the insoluble cellulose material and the different enzymes. In 2010 Quirk et al. reported the first study on the direct visualization of the process of biochemical cellulose depolymerization using AFM.(156) This study used Acetobacter xylinum crystalline cellulose fibers as the cellulosic substrate and C. fimi CenA endoglucanase as the depolymerizing glycoside hydrolase. The depolymerization was observed at room temperature in real time over nine hours and AFM images of the fibrils as they were degraded were obtained in both topography and amplitude mode. Quirk et al. observed that over the experimental time frame cellulose fibrils were degraded preferentially at the ends of fibrils, as well as at the sites where the cellulose fibres exhibited defects and kinks. The visual result of the depolymerization was progressive fraying and shortening of the fibre ends. In addition, they were able to observe significant swelling of the fibers at sites where no visible degradation occurred, which was interpreted as the disruption of the crystalline packing of the cellulose by CenA cellulases. The same year, Santa-Maria and Jeoh reported a detailed study of the depolymerization of crystalline cellulose by a purified T. reseii cellobiohydrolase (Cel7A), 
which combined AFM and confocal fluorescence microscopy imaging.(157) In this study, the authors conducted AFM and confocal imaging of bacterial and algal cellulose that had been degraded in batch preparations, as well real time imaging of cellulose that was degraded in situ. They observed that during the initial degradation the cellulose microfibrils experienced untwisting, which could be interpreted as the relaxation of the inherent internal stress of chiral cellulose crystals. This study also revealed that during degradation the cellulose microfibrils shortened and thinned out, with 0.3-0.6 nm grooves becoming evident along the length of the microfibrils at large extents of hydrolysis $(80 \%)$. The appearance of such channels was attributed to the processive action of Cel7A cellobiohydrolase, where the hydrolysis of a single glucan chain leaves a void behind. This report was the first to visualize the tension release as a result of cellulose hydrolysis.

Fluorescence microscopy techniques have also been recently applied to study the hydrolysis of cellulosic materials. The capability of fluorescence techniques to monitor individual species within a complex mixture of proteins has made these techniques applicable to the study of cellulose degradation by mixtures of cellulolytic enzymes. Thygesen and collaborators have used polarized light microscopy together with confocal microscopy to study the degradation of filter paper and pretreated wheat straw cellulosic fibres by commercial cellulolytic cocktails.(158) In this study, the authors focused on characterizing the changes occurring at dislocations present in the cellulosic fibres and used a fluorescently labeled cellulase (endoglucanase GH45 from Humicola insolens) as a reporter for cellulase binding and hydrolysis. They observed that the dislocations observed in the cellulose fibres were not completely amorphous, as they exhibited the characteristic birefringence of crystalline cellulose. Such dislocations were shown to be the primary site of binding for the fluorescent endoglucanases. It was hypothesized that the dislocations are sites with higher accessibility, which leads to the preferential binding and more rapid hydrolysis. The hypothesis that dislocations are the sites for the initiation of fibre hydrolysis was further supported by the observation that as hydrolysis progresses, the fibres remaining in the sample were shorter and the number of dislocations observed was reduced. This study showed that dislocations play an important role during the initial stages of hydrolysis of lignocellulose. More recently, Luterbacher et al. also used confocal fluorescence microscopy to monitor the degradation of bacterial microcrystalline cellulose using fluorescent $T$. reseii Cel7A cellobiohydrolase as a reporter doped within a commercial cellulolytic cocktail.(159) In this study the authors completely hydrolyzed the cellulosic substrate and used quantitative fluorescence intensity measurements to determine the degree of hydrolysis achieved. They correlated the observed fluorescence intensity with remaining cellulose and were able to fit a theoretical model to the observed fluorescence. The kinetic parameters obtained from these fits were able to correctly predict the total sugars released from the cellulose and compared favourably with values obtained through degradation experiments conducted in bulk. In addition, the kinetic parameters obtained pointed to the availability of cellulase binding sites as a key limiting factor for the degradation of crystalline cellulose. This study demonstrated for the first time that quantitative fluorescence techniques could be 
used to monitor degradation of cellulosic substrates and extract relevant degradation kinetics.

\section{Concluding remarks and future outlook}

Advanced microscopy techniques have enabled the acquisition of images of cellulosic substrates with higher spatial and temporal resolution. Both scanning probe microscopy and fluorescence microscopy techniques have already been applied to image the inherent structure of cellulosic materials, such as in the distribution of structural polysaccharides within the cell wall or the arrangement of glucan chains within crystalline cellulose fibrils; the interaction of glycoside hydrolases with cellulosic substrates, such as in the binding, diffusion and hydrolytic activity of cellulases and their cellulose binding domains; and the structural changes that cellulosic materials undergo as they are subjected to different chemical, physical and biochemical treatments. However, new imaging modalities, especially those that exploit the ability to image and track single molecules, are just starting to be applied to the study of cellulose. It is anticipated that such techniques, as they become more widespread, will be of increasing importance to scientists interested in extracting information at the nanometer length scale where the accuracy of localization and the concomitant resolution approximate the molecular size of the basic units of structural polysaccharides and the size of individual glycoside hydrolases. In this way, the application of Fast Scan Atomic Force Microscopy or Single Molecule Fluorescence Microscopy could evidence real time behaviour of the different hydrolases as they interact with cellulose microfibrils. Furthermore, single molecule techniques could probe the enzyme activity and determine the fraction of glycoside hydrolases adsorbed to cellulose chains that are in a catalytic active state. Single molecule measurements could also reveal heterogeneities in the binding of cellulases to different structural motifs within the cell wall. All these measurements could provide further insight into the mechanistic action of the enzymes that depolymerize lignocellulosic materials and could help understand the inactivation of enzymes that occurs during the saccharification process. The ability of fluorescence techniques to monitor multiple species simultaneously could also provide insight into the synergistic action of glycoside hydrolases that are incorporated into cellulolytic cocktails. Other advanced microscopy techniques, such as Super-Resolution Fluorescence Microscopy, will undoubtedly contribute to understanding the changes that lignocellulosic materials undergo during different pretreatments by providing nanoscale resolution images of one or more of the structural polysaccharides within the cell walls of plant biomass. These advances taken together as a whole will further our understanding of how the cell wall is constructed and how to more efficiently harness the richness of plant biomass to produce fuels, plastics and other chemicals in a more sustainable, environmentally friendly, and economically viable fashion. The study of cellulose through advanced microscopy techniques is in its nascent stages and many exciting opportunities exist to develop and apply experimental methods that can offer a glimpse on the nanoscale behaviour of cellulosic materials with high temporal resolution. 


\section{Author details}

Jose M. Moran-Mirabal

Department of Chemistry and Chemical Biology, McMaster University, Hamilton, ON, Canada

\section{References}

[1] Lee KJ., Marcus SE, Knox JP. Cell wall biology: perspectives from cell wall imaging. Molecular plant. 2011;4(2):212-9.

[2] Chundawat SP., Beckham GT, Himmel ME, Dale BE. Deconstruction of lignocellulosic biomass to fuels and chemicals. Annual Review of Chemical and Biomolecular Engineering. 2011;2:121-45.

[3] Pauly M, Keegstra K. Cell-wall carbohydrates and their modification as a resource for biofuels. The Plant Journal. 2008;54(4):559-68.

[4] Yarbrough JM, Himmel ME, Ding SY. Plant cell wall characterization using scanning probe microscopy techniques. Biotechnology for biofuels. 2009;2(1):17.

[5] Rubin EM. Genomics of cellulosic biofuels. Nature. 2008 Aug;454(7206):841-5.

[6] MCCANN MC, WELLS B, ROBERTS K. Direct visualization of cross-links in the primary plant cell wall. Journal of Cell Science. 1990 Jun 1;96(2):323 -334.

[7] Carpita N, Sabularse D, Montezinos D, Delmer DP. Determination of the Pore Size of Cell Walls of Living Plant Cells. Science. 1979 Sep 14;205(4411):1144-7.

[8] Grethlein HE. The Effect of Pore Size Distribution on the Rate of Enzymatic Hydrolysis of Cellulosic Substrates. Nat Biotechnol. 1985 Feb;3(2):155-60.

[9] Fengel D, Stoll M. Crystals of cellulose grown from TFA solution. Wood Science and Technology. 1989;23(1):85-94.

[10] Riley R, Gardner JO, Merten U. Cellulose acetate membranes: Electron microscopy of structure. Science. 1964;143(3608):801-3.

[11] Cronshaw J, Myers A, Preston RD. A chemical and physical investigation of the cell walls of some marine algae. Biochimica et Biophysica Acta. 1958 Jan;27:89-103.

[12] Carpita NC, Defernez M, Findlay K, Wells B, Shoue DA, Catchpole G, et al. Cell wall architecture of the elongating maize coleoptile. Plant physiology. 2001;127(2):551-65.

[13] Henriksson M, Berglund LA, Isaksson P, Lindström T, Nishino T. Cellulose Nanopaper Structures of High Toughness. Biomacromolecules. 2008 Jun;9(6):1579-85.

[14] Kvien I, Tanem BS, Oksman K. Characterization of Cellulose Whiskers and Their Nanocomposites by Atomic Force and Electron Microscopy. Biomacromolecules. 2005 Nov;6(6):3160-5.

[15] O'SULLIVAN AC. Cellulose: the structure slowly unravels. Cellulose. 1997;4(3):173207.

[16] Turner SR, Somerville CR. Collapsed xylem phenotype of Arabidopsis identifies mutants deficient in cellulose deposition in the secondary cell wall. The Plant Cell Online. 1997;9(5):689-701. 
[17] Revol JF, Bradford H, Giasson J, Marchessault RH, Gray DG. Helicoidal self-ordering of cellulose microfibrils in aqueous suspension. International journal of biological macromolecules. 1992;14(3):170-2.

[18] Heyn ANJ. The microcrystalline structure of cellulose in cell walls of cotton, ramie, and ute fibers as revealed by negative staining of sections. The Journal of cell biology. 1966;29(2):181-97.

[19] White AR, Brown RM. Enzymatic hydrolysis of cellulose: Visual characterization of the process. Proceedings of the National Academy of Sciences. 1981;78(2):1047.

[20] Hult EL, Iversen T, Sugiyama J. Characterization of the supermolecular structure of cellulose in wood pulp fibres. Cellulose. 2003;10(2):103-10.

[21] Fotiadis D, Scheuring S, Müller SA, Engel A, Müller DJ. Imaging and manipulation of biological structures with the AFM. Micron. 2002;33(4):385-97.

[22] Israelachvili JN. Intermolecular and surface forces. Burlington, MA: Academic Press; 2011.

[23] Deniz AA, Mukhopadhyay S, Lemke EA. Single-molecule biophysics: at the interface of biology, physics and chemistry. Journal of The Royal Society Interface. 2008;5(18):15-45.

[24] Ando T, Uchihashi T, Kodera N, Yamamoto D, Miyagi A, Taniguchi M, et al. Highspeed AFM and nano-visualization of biomolecular processes. Pflügers Archiv European Journal of Physiology. 2007 Dec 20;456(1):211-25.

[25] Ando T, Kodera N, Naito Y, Kinoshita T, Furuta K, Toyoshima YY. A High-speed Atomic Force Microscope for Studying Biological Macromolecules in Action. ChemPhysChem. 2003 Nov 14;4(11):1196-202.

[26] Ando T, Uchihashi T, Kodera N, Miyagi A, Nakakita R, Yamashita H, et al. High-Speed Atomic Force Microscopy for Studying the Dynamic Behavior of Protein Molecules at Work. Japanese Journal of Applied Physics. 2006 Mar 27;45(3B):1897-903.

[27] Igarashi K, Koivula A, Wada M, Kimura S, Penttila M, Samejima M. High Speed Atomic Force Microscopy Visualizes Processive Movement of Trichoderma reesei Cellobiohydrolase I on Crystalline Cellulose. Journal of Biological Chemistry. 2009 Oct 26;284(52):36186-90.

[28] Igarashi K, Uchihashi T, Koivula A, Wada M, Kimura S, Okamoto T, et al. Traffic Jams Reduce Hydrolytic Efficiency of Cellulase on Cellulose Surface. Science. 2011 Sep 1;333(6047):1279-82.

[29] Lakowicz JR. Principles of Fluorescence Spectroscopy. New York, USA: Kluwer Academic/Plenum; 1999.

[30] Shaner NC, Steinbach PA, Tsien RY. A guide to choosing fluorescent proteins. Nature Methods. 2005 Dec;2(12):905-9.

[31] Giepmans BNG. The Fluorescent Toolbox for Assessing Protein Location and Function. Science. 2006 Apr 14;312(5771):217-24.

[32] Tsien RY. THE GREEN FLUORESCENT PROTEIN. Annual Review of Biochemistry. 1998 Jun;67(1):509-44. 
[33] Kino GS, Corle TR. Confocal Scanning Optical Microscopy. Physics Today. 1989;42(9):55.

[34] Xiao GQ, Corle TR, Kino GS. Real-time confocal scanning optical microscope. Applied Physics Letters. 1988;53(8):716.

[35] Corle TR, Chou C-H, Kino GS. Depth response of confocal optical microscopes. Optics Letters. 1986 Dec 1;11(12):770.

[36] Axelrod D, Burghardt TP, Thompson NL. Total Internal Reflection Fluorescence. Annual Review of Biophysics and Bioengineering. 1984 Jun;13(1):247-68.

[37] Ambrose WP, Goodwin PM, Nolan JP. Single-molecule detection with total internal reflection excitation: Comparing signal-to-background and total signals in different geometries. Cytometry. 1999 Jul 1;36(3):224-31.

[38] Axelrod D. Selective imaging of surface fluorescence with very high aperture microscope objectives. Journal of Biomedical Optics. 2001;6(1):6.

[39] Paige MF, Bjerneld EJ, Moerner WE. A Comparison of Through-the-Objective Total Internal Reflection Microscopy and Epifluorescence Microscopy for Single-Molecule Fluorescence Imaging. Single Molecules. 2001 Oct;2(3):191-201.

[40] Axelrod D. Cell-substrate contacts illuminated by total internal reflection fluorescence. The Journal of Cell Biology. 1981 Apr 1;89(1):141-5.

[41] Axelrod D. Total Internal Reflection Fluorescence Microscopy in Cell Biology. Traffic. 2001 Nov;2(11):764-74.

[42] Axelrod D, Koppel D, Schlessinger J, Elson E, Webb W. Mobility measurement by analysis of fluorescence photobleaching recovery kinetics. Biophysical Journal. 1976 Sep;16(9):1055-69.

[43] Sprague BL, Pego RL, Stavreva DA, McNally JG. Analysis of binding reactions by fluorescence recovery after photobleaching. Biophysical Journal. 2004;86(6):3473-95.

[44] Sprague B, McNally J. FRAP analysis of binding: proper and fitting. Trends in Cell Biology. 2005 Feb;15(2):84-91.

[45] Sund SE, Axelrod D. Actin dynamics at the living cell submembrane imaged by total internal reflection fluorescence photobleaching. Biophysical Journal. 2000;79(3):1655-69.

[46] Cole NB, Smith CL, Sciaky N, Terasaki M, Edidin M, Lippincott-Schwartz J. Diffusional Mobility of Golgi Proteins in Membranes of Living Cells. Science. 1996 Aug;273(5276):797-801.

[47] Tirnauer JS, Grego S, Salmon ED, Mitchison TJ. EB1-microtubule interactions in Xenopus egg extracts: role of EB1 in microtubule stabilization and mechanisms of targeting to microtubules. Molecular biology of the cell. 2002;13(10):3614.

[48] Stenoien DL, Patel K, Mancini MG, Dutertre M, Smith CL, O'Malley BW, et al. FRAP reveals that mobility of oestrogen receptor-\$ $\backslash$ alpha $\$$ is ligand-and proteasomedependent. Nature cell biology. 2000;3(1):15-23.

[49] Mochizuki N, Yamashita S, Kurokawa K, Ohba Y, Nagai T, Miyawaki A, et al. Spatiotemporal images of growth-factor-induced activation of Ras and Rap1. Nature. 2001;411(6841):1065-8. 
[50] Stryer L. Energy Transfer: A Spectroscopic Ruler. Proceedings of the National Academy of Sciences. 1967 Aug 1;58(2):719-26.

[51] Jares-Erijman EA, Jovin TM. FRET imaging. Nature Biotechnology. 2003 Nov;21(11):1387-95.

[52] Kapanidis AN, Weiss S. Fluorescent probes and bioconjugation chemistries for singlemolecule fluorescence analysis of biomolecules. The Journal of Chemical Physics. 2002;117(24):10953.

[53] Clegg RM. Fluorescence resonance energy transfer. Current Opinion in Biotechnology. 1995 Jan;6(1):103-10.

[54] Ha T. Single-Molecule Fluorescence Resonance Energy Transfer. Methods. 2001 Sep;25(1):78-86.

[55] Selvin PR, others. The renaissance of fluorescence resonance energy transfer. nature structural biology. 2000;7(9):730-4.

[56] Sekar RB. Fluorescence resonance energy transfer (FRET) microscopy imaging of live cell protein localizations. The Journal of Cell Biology. 2003 Mar 3;160(5):629-33.

[57] Chen Y, Müller JD, So PTC, Gratton E. The Photon Counting Histogram in Fluorescence Fluctuation Spectroscopy. Biophysical Journal. 1999 Jul;77(1):553-67.

[58] Puchalla J, Krantz K, Austin R, Rye H. Burst analysis spectroscopy: A versatile singleparticle approach for studying distributions of protein aggregates and fluorescent assemblies. Proceedings of the National Academy of Sciences. 2008 Sep 5;105(38):144005.

[59] Stavis SM, Corgié SC, Cipriany BR, Craighead HG, Walker LP. Single molecule analysis of bacterial polymerase chain reaction products in submicrometer fluidic channels. Biomicrofluidics. 2007;1(3):034105.

[60] Stavis SM, Edel JB, Samiee KT, Craighead HG. Single molecule studies of quantum dot conjugates in a submicrometer fluidic channel. Lab on a Chip. 2005;5(3):337.

[61] Bacia K, Kim SA, Schwille P. Fluorescence cross-correlation spectroscopy in living cells. Nature Methods. 2006 Feb;3(2):83-9.

[62] Bacia K, Schwille P. A dynamic view of cellular processes by in vivo fluorescence autoand cross-correlation spectroscopy. Methods. 2003 Jan;29(1):74-85.

[63] Haustein E. Ultrasensitive investigations of biological systems by fluorescence correlation spectroscopy. Methods. 2003 Feb;29(2):153-66.

[64] Haustein E, Schwille P. Single-molecule spectroscopic methods. Current Opinion in Structural Biology. 2004 Oct;14(5):531-40.

[65] Rigler R, Földes-Papp Z, Meyer-Almes F-J, Sammet C, Völcker M, Schnetz A. Fluorescence cross-correlation: A new concept for polymerase chain reaction. Journal of Biotechnology. 1998 Aug;63(2):97-109.

[66] Schwille P, Meyer-Almes FJ, Rigler R. Dual-color fluorescence cross-correlation spectroscopy for multicomponent diffusional analysis in solution. Biophysical Journal. 1997 Apr;72(4):1878-86. 
[67] Webb WW. Fluorescence Correlation Spectroscopy: Inception, Biophysical Experimentations, and Prospectus. Applied Optics. 2001;40(24):3969.

[68] Carter BC, Shubeita GT, Gross SP. Tracking single particles: a user-friendly quantitative evaluation. Physical Biology. 2005 Mar;2(1):60-72.

[69] Cheezum MK, Walker WF, Guilford WH. Quantitative Comparison of Algorithms for Tracking Single Fluorescent Particles. Biophysical Journal. 2001 Oct;81(4):2378-88.

[70] Ghosh RN, Webb WW. Automated detection and tracking of individual and clustered cell surface low density lipoprotein receptor molecules. Biophysical Journal. 1994 May;66(5):1301-18.

[71] Basché T, Ambrose WP, Moerner WE. Optical spectra and kinetics of single impurity molecules in a polymer: spectral diffusion and persistent spectral hole burning. Journal of the Optical Society of America B. 1992 May 1;9(5):829.

[72] Jaqaman K, Loerke D, Mettlen M, Kuwata H, Grinstein S, Schmid SL, et al. Robust single-particle tracking in live-cell time-lapse sequences. Nature Methods. $2008 \mathrm{Jul}$ 20;5(8):695-702.

[73] Yildiz A. Myosin V Walks Hand-Over-Hand: Single Fluorophore Imaging with 1.5-nm Localization. Science. 2003 Jun 27;300(5628):2061-5.

[74] Lang E, Baier J, Kohler J. Epifluorescence, confocal and total internal reflection microscopy for single-molecule experiments: a quantitative comparison. J Microsc. 2006 May;222(2):118-23.

[75] Greenleaf WJ, Woodside MT, Block SM. High-Resolution, Single-Molecule Measurements of Biomolecular Motion. Annual Review of Biophysics and Biomolecular Structure. 2007 Jun;36(1):171-90.

[76] Ha T. Single-Molecule Fluorescence Resonance Energy Transfer. Methods. 2001 Sep;25(1):78-86.

[77] Hess ST, Huang S, Heikal AA, Webb WW. Biological and Chemical Applications of Fluorescence Correlation Spectroscopy: A Review t. Biochemistry. 2002 Jan;41(3):697705.

[78] Joo C, Balci H, Ishitsuka Y, Buranachai C, Ha T. Advances in Single-Molecule Fluorescence Methods for Molecular Biology. Annual Review of Biochemistry. 2008 Jun;77(1):51-76.

[79] Michalet X, Weiss S, Jäger M. Single-Molecule Fluorescence Studies of Protein Folding and Conformational Dynamics. Chemical Reviews. 2006 May;106(5):1785-813.

[80] Moerner WE. Single-molecule Chemistry and Biology Special Feature: New directions in single-molecule imaging and analysis. Proceedings of the National Academy of Sciences. 2007 May 11;104(31):12596-602.

[81] Moerner WE, Fromm DP. Methods of single-molecule fluorescence spectroscopy and microscopy. Review of Scientific Instruments. 2003;74(8):3597.

[82] Nie S, Zare RN. OPTICAL DETECTION OF SINGLE MOLECULES. Annual Review of Biophysics and Biomolecular Structure. 1997 Jun;26(1):567-96. 
[83] Tinnefeld P, Sauer M. Branching Out of Single-Molecule Fluorescence Spectroscopy: Challenges for Chemistry and Influence on Biology. Angewandte Chemie International Edition. 2005 Apr 29;44(18):2642-71.

[84] Weiss S. Fluorescence Spectroscopy of Single Biomolecules. Science. 1999 Mar 12;283(5408):1676-83.

[85] Weiss S, others. Measuring conformational dynamics of biomolecules by single molecule fluorescence spectroscopy. Nature structural biology. 2000;7(9):724-9.

[86] Xie XS, Trautman JK. OPTICAL STUDIES OF SINGLE MOLECULES AT ROOM TEMPERATURE. Annual Review of Physical Chemistry. 1998 Oct;49(1):441-80.

[87] Schermelleh L, Carlton PM, Haase S, Shao L, Winoto L, Kner P, et al. Subdiffraction Multicolor Imaging of the Nuclear Periphery with 3D Structured Illumination Microscopy. Science. 2008 Jun;320(5881):1332-6.

[88] Hell SW. Toward fluorescence nanoscopy. Nat Biotechnol. 2003 Nov;21(11):1347-55.

[89] Gustafsson MG. Nonlinear structured-illumination microscopy: Wide-field fluorescence imaging with theoretically unlimited resolution. Proceedings of the National Academy of Sciences. 2005;102(37):13081.

[90] Nagerl UV, Willig KI, Hein B, Hell SW, Bonhoeffer T. Live-cell imaging of dendritic spines by STED microscopy. Proceedings of the National Academy of Sciences. 2008 Nov;105(48):18982-7.

[91] Gustafsson MG. Extended resolution fluorescence microscopy. Current opinion in structural biology. 1999;9(5):627-8.

[92] Shtengel G, Galbraith JA, Galbraith CG, Lippincott-Schwartz J, Gillette JM, Manley S, et al. Interferometric fluorescent super-resolution microscopy resolves 3D cellular ultrastructure. Proceedings of the National Academy of Sciences. 2009 Feb;106(9):312530.

[93] Manley S, Gillette JM, Patterson GH, Shroff H, Hess HF, Betzig E, et al. High-density mapping of single-molecule trajectories with photoactivated localization microscopy. Nat Meth. 2008 Jan;5(2):155-7.

[94] Bates M, Huang B, Zhuang X. Super-resolution microscopy by nanoscale localization of photo-switchable fluorescent probes. Current Opinion in Chemical Biology. 2008 Oct;12(5):505-14.

[95] Cordes T, Strackharn M, Stahl SW, Summerer W, Steinhauer C, Forthmann C, et al. Resolving Single-Molecule Assembled Patterns with Superresolution Blink-Microscopy. Nano Lett. 2010 Feb;10(2):645-51.

[96] Heilemann M, van de Linde S, Schüttpelz M, Kasper R, Seefeldt B, Mukherjee A, et al. Subdiffraction-Resolution Fluorescence Imaging with Conventional Fluorescent Probes. Angew. Chem. Int. Ed. 2008 Aug;47(33):6172-6.

[97] Vogelsang J, Cordes T, Forthmann C, Steinhauer C, Tinnefeld P. Controlling the fluorescence of ordinary oxazine dyes for single-molecule switching and superresolution microscopy. Proceedings of the National Academy of Sciences. 2009;106(20):8107. 
[98] Huang B, Wang W, Bates M, Zhuang X. Three-Dimensional Super-Resolution Imaging by Stochastic Optical Reconstruction Microscopy. Science. 2008 Feb;319(5864):810-3.

[99] Heintzmann R, Ficz G. Breaking the resolution limit in light microscopy. Briefings in Functional Genomics and Proteomics. 2006 May;5(4):289-301.

[100] Lippincott-Schwartz J, Manley S. Putting super-resolution fluorescence microscopy to work. Nat Meth. 2009 Jan;6(1):21-3.

[101] Moran-Mirabal JM. Study of cellulase-cellulose interactions through fluorescence microscopy techniques. Cellulose. 2013; In Press.

[102] Fernandes AN, Thomas LH, Altaner CM, Callow P, Forsyth VT, Apperley DC, et al. Nanostructure of cellulose microfibrils in spruce wood. Proceedings of the National Academy of Sciences. 2011;108(47):E1195-E1203.

[103] Kirby AR, Gunning AP, Waldron KW, Morris VJ, Ng A. Visualization of plant cell walls by atomic force microscopy. Biophysical Journal. 1996 Mar;70(3):1138-43.

[104] Pesacreta TC, Carlson LC, Triplett BA. Atomic force microscopy of cotton fiber cell wall surfaces in air and water: quantitative and qualitative aspects. Planta. 1997;202(4):435-42.

[105] Thimm JC, Burritt DJ, Ducker WA, Melton LD. Celery (Apium graveolens L.) parenchyma cell walls examined by atomic force microscopy: effect of dehydration on cellulose microfibrils. Planta. 2000;212(1):25-32.

[106] Davies LM, Harris PJ. Atomic force microscopy of microfibrils in primary cell walls. Planta. 2003;217(2):283-9.

[107] Ding S-Y, Himmel ME. The Maize Primary Cell Wall Microfibril: A New Model Derived from Direct Visualization. J. Agric. Food Chem. 2006 Feb;54(3):597-606.

[108] Sugiyama J, Harada H, Fujiyoshi Y, Uyeda N. Lattice images from ultrathin sections of cellulose microfibrils in the cell wall of Valonia macrophysa Kütz. Planta. 1985;166(2):161-8.

[109] Hanley SJ, Giasson J, Revol J-F, Gray DG. Atomic force microscopy of cellulose microfibrils: comparison with transmission electron microscopy. Polymer. 1992;33(21):4639-42.

[110] Baker AA, Helbert W, Sugiyama J, Miles MJ. High-Resolution Atomic Force Microscopy of NativeValoniaCellulose I Microcrystals. Journal of Structural Biology. 1997 Jul;119(2):129-38.

[111] Baker AA, Helbert W, Sugiyama J, Miles MJ. Surface structure of native cellulose microcrystals by AFM. Applied Physics A: Materials Science \& Processing. 1998;66:55963.

[112] Baker AA, Helbert W, Sugiyama J, Miles MJ. New Insight into Cellulose Structure by Atomic Force Microscopy Shows the I [alpha] Crystal Phase at Near-Atomic Resolution. Biophysical Journal. 2000;79(2):1139-45.

[113] Koljonen K, Österberg M, Johansson LS, Stenius P. Surface chemistry and morphology of different mechanical pulps determined by ESCA and AFM. Colloids and Surfaces A: Physicochemical and Engineering Aspects. 2003;228(1-3):143-58. 
[114] Gustafsson J, Ciovica L, Peltonen J. The ultrastructure of spruce kraft pulps studied by atomic force microscopy (AFM) and X-ray photoelectron spectroscopy (XPS). Polymer. 2003;44(3):661-70.

[115] Fahlen J, Salmen L. Cross-sectional structure of the secondary wall of wood fibers as affected by processing. Journal of materials science. 2003;38(1):119-26.

[116] Schmied FJ, Teichert C, Kappel L, Hirn U, Schennach R. Analysis of precipitated lignin on kraft pulp fibers using atomic force microscopy. Cellulose. 2012 Feb 18;19(3):1013-21.

[117] Eronen P, Österberg M, Jääskeläinen A-S. Effect of alkaline treatment on cellulose supramolecular structure studied with combined confocal Raman spectroscopy and atomic force microscopy. Cellulose. 2008 Oct 11;16(2):167-78.

[118] Kristensen JB, Thygesen LG, Felby C, Jørgensen H, Elder T, others. Cell-wall structural changes in wheat straw pretreated for bioethanol production. Biotechnol Biofuels. 2008;1(5):1-9.

[119] Pang C, Xie T, Lin L, Zhuang J, Liu Y, Shi J, et al. Changes of the surface structure of corn stalk in the cooking process with active oxygen and MgO-based solid alkali as a pretreatment of its biomass conversion. Bioresource Technology. 2012 Jan;103(1):432-9.

[120] Kontturi E, Vuorinen T. Indirect evidence of supramolecular changes within cellulose microfibrils of chemical pulp fibers upon drying. Cellulose. 2008 Jun 12;16(1):65-74.

[121] Sèbe G, Ham-Pichavant F, Ibarboure E, Koffi ALC, Tingaut P. Supramolecular structure characterization of cellulose II nanowhiskers produced by acid hydrolysis of cellulose I substrates. Biomacromolecules [Internet]. 2012 [cited 2012 Jul 6]; Available from: http://pubs.acs.org/doi/abs/10.1021/bm201777j

[122] Hayashi T, Maclachlan G. Pea xyloglucan and cellulose: I. Macromolecular organization. Plant Physiol. 1984;75(3):596.

[123] Brunecky R, Vinzant TB, Porter SE, Donohoe BS, Johnson DK, Himmel ME. Redistribution of xylan in maize cell walls during dilute acid pretreatment. Biotechnology and Bioengineering. 2009 Apr 15;102(6):1537-43.

[124] Sandquist D, Filonova L, von Schantz L, Ohlin M, Daniel G. Microdistribution of xyloglucan in differentiating poplar cells. Bio. Res. 2010;5(2):796-807.

[125] Arend M. Immunolocalization of $(1,4)-\$ \backslash$ beta-galactan in tension wood fibers of poplar. Tree Physiology. 2008;28(8):1263-7.

[126] Boraston AB, Bolam DN, Gilbert HJ, Davies GJ. Carbohydrate-binding modules: finetuning polysaccharide recognition. Biochemical Journal. 2004 Sep 15;382(3):769.

[127] Cantarel BL, Coutinho PM, Rancurel C, Bernard T, Lombard V, Henrissat B. The Carbohydrate-Active EnZymes database (CAZy): an expert resource for Glycogenomics. Nucleic Acids Research. 2009 Jan 1;37(Database):D233-D238.

[128] Linder M, Winiecka-Krusnell J, Linder E. Use of Recombinant Cellulose-Binding Domains of Trichoderma reesei Cellulase as a Selective Immunocytochemical Marker for Cellulose in Protozoa. Applied and Environmental Microbiology. 2002 May 1;68(5):2503-8. 
[129] McCartney L, Gilbert HJ, Bolam DN, Boraston AB, Knox JP. Glycoside hydrolase carbohydrate-binding modules as molecular probes for the analysis of plant cell wall polymers. Analytical Biochemistry. 2004 Mar;326(1):49-54.

[130] McCartney L, Blake AW, Flint J, Bolam DN, Boraston AB, Gilbert HJ, et al. Differential recognition of plant cell walls by microbial xylan-specific carbohydrate-binding modules. Proceedings of the National Academy of Sciences of the United States of America. 2006;103(12):4765-70.

[131] Daniel G, Filonova L, Kallas ÅM, Teeri TT. Morphological and chemical characterisation of the G-layer in tension wood fibres of Populus tremula and Betula verrucosa: Labelling with cellulose-binding module CBM1 $\mathrm{Hj}$ Cel7A and fluorescence and FE-SEM microscopy. Holzforschung. 2006 Jan 1;60(6):618-24.

[132] Široký J, Benians TAS, Russell SJ, Bechtold T, Paul Knox J, Blackburn RS. Analysis of crystallinity changes in cellulose II polymers using carbohydrate-binding modules. Carbohydrate Polymers. 2012 Jun;89(1):213-21.

[133] Filonova L, Gunnarsson L, Daniel G, Ohlin M. Synthetic xylan-binding modules for mapping of pulp fibres and wood sections. BMC Plant Biology. 2007;7(1):54.

[134] Zhang P. Investigation of Novel Quantum Dots/Proteins/Cellulose Bioconjugate Using NSOM and Fluorescence. Journal of Fluorescence. 2006 Feb 14;16(3):349-53.

[135] Xu Q, Tucker MP, Arenkiel P, Ai X, Rumbles G, Sugiyama J, et al. Labeling the planar face of crystalline cellulose using quantum dots directed by type-I carbohydratebinding modules. Cellulose. 2008 Jun 11;16(1):19-26.

[136] Ding S-Y, Xu Q, Ali M, Baker J, Bayer E, Barak Y, et al. Versatile derivatives of carbohydrate-binding modules for imaging of complex carbohydrates approaching the molecular level of resolution. BioTechniques. 2006 Oct;41(4):435-43.

[137] Porter SE, Donohoe BS, Beery KE, Xu Q, Ding S-Y, Vinzant TB, et al. Microscopic analysis of corn fiber using starch- and cellulose-specific molecular probes. Biotechnology and Bioengineering. 2007 Sep 1;98(1):123-31.

[138] Kawakubo T, Karita S, Araki Y, Watanabe S, Oyadomari M, Takada R, et al. Analysis of exposed cellulose surfaces in pretreated wood biomass using carbohydrate-binding module (CBM)â cyan fluorescent protein (CFP). Biotechnology and Bioengineering. 2010 Feb 15;105(3):499-508.

[139] Lacayo CI, Malkin AJ, Holman H-YN, Chen L, Ding S-Y, Hwang MS, et al. Imaging Cell Wall Architecture in Single Zinnia elegans Tracheary Elements. PLANT PHYSIOLOGY. 2010 Jun 30;154(1):121-33.

[140] Umemoto Y, Araki T. Cell Wall Regeneration in Bangia atropurpurea (Rhodophyta) Protoplasts Observed Using a Mannan-Specific Carbohydrate-Binding Module. Marine Biotechnology. 2009 May 23;12(1):24-31.

[141] Dagel DJ, Liu Y-S, Zhong L, Luo Y, Himmel ME, Xu Q, et al. In Situ Imaging of Single Carbohydrate-Binding Modules on Cellulose Microfibrils. J. Phys. Chem. B. 2011 Feb;115(4):635-41. 
[142] Fox JM, Jess P, Jambusaria RB, Moo GM, Liphardt J, Clark DS, et al. A single-molecule analysis reveals morphological targets for cellulase synergy. Nature Chemical Biology [Internet]. 2013 Apr 7 [cited 2013 Apr 23]; Available from: http://www.nature.com/doifinder/10.1038/nchembio.1227

[143] Pinto R, Carvalho J, Mota M, Gama M. Large-scale production of cellulose-binding domains. Adsorption studies using CBD-FITC conjugates. Cellulose. 2006 Apr 12;13(5):557-69.

[144] Pinto R, Amaral AL, Carvalho J, Ferreira EC, Mota M, Gama M. Development of a Method Using Image Analysis for the Measurement of Cellulose-Binding Domains Adsorbed onto Cellulose Fibers. Biotechnology Progress. 2007 Dec 7;23(6):1492-7.

[145] Pinto R, Amaral AL, Ferreira EC, Mota M, Vilanova M, Ruel K, et al. Quantification of the CBD-FITC conjugates surface coating on cellulose fibres. BMC Biotechnology. 2008;8(1):1.

[146] Moran-Mirabal JM, Santhanam N, Corgie SC, Craighead HG, Walker LP. Immobilization of cellulose fibrils on solid substrates for cellulase-binding studies through quantitative fluorescence microscopy. Biotechnology and Bioengineering. 2008 Dec 15;101(6):1129-41.

[147] Zhu P, Moran-Mirabal JM, Luterbacher JS, Walker LP, Craighead HG. Observing Thermobifida fusca cellulase binding to pretreated wood particles using time-lapse confocal laser scanning microscopy. Cellulose. 2011 Feb 6;18(3):749-58.

[148] Wang L, Wang Y, Ragauskas AJ. A novel FRET approach for in situ investigation of cellulase-cellulose interaction. Analytical and Bioanalytical Chemistry. 2010 Aug 8;398(3):1257-62.

[149] Wang L, Wang Y, Ragauskas AJ. Determination of cellulase colocalization on cellulose fiber with quantitative FRET measured by acceptor photobleaching and spectrally unmixing fluorescence microscopy. The Analyst. 2012;137(6):1319.

[150] Moran-Mirabal JM, Bolewski JC, Walker LP. Reversibility and binding kinetics of Thermobifida fusca cellulases studied through fluorescence recovery after photobleaching microscopy. Biophysical Chemistry. 2011 Apr;155(1):20-8.

[151] Jervis EJ, Haynes CA, Kilburn DG. Surface diffusion of cellulases and their isolated binding domains on cellulose. Journal of Biological Chemistry. 1997;272(38):24016.

[152] Cuyvers S, Hendrix J, Dornez E, Engelborghs Y, Delcour JA, Courtin CM. Both Substrate Hydrolysis and Secondary Substrate Binding Determine Xylanase Mobility as Assessed by FRAP. The Journal of Physical Chemistry B. 2011 Apr 28;115(16):4810-7.

[153] Moran-Mirabal JM, Bolewski JC, Walker LP. Thermobifida fusca cellulases exhibit limited surface diffusion on bacterial micro-crystalline cellulose. Biotechnology and Bioengineering. 2013 Jan;110(1):47-56.

[154] Horvath AT, Horvath AE, Lindström T, Wågberg L. Diffusion of Cationic Polyelectrolytes into Cellulosic Fibers. Langmuir. 2008 Oct 7;24(19):10797-806.

[155] Himmel ME, Ding S-Y, Johnson DK, Adney WS, Nimlos MR, Brady JW, et al. Biomass Recalcitrance: Engineering Plants and Enzymes for Biofuels Production. Science. 2007 Feb;315(5813):804-7. 
[156] Quirk A, Lipkowski J, Vandenende C, Cockburn D, Clarke AJ, Dutcher JR, et al. Direct Visualization of the Enzymatic Digestion of a Single Fiber of Native Cellulose in an Aqueous Environment by Atomic Force Microscopy. Langmuir. 2010 Apr 6;26(7):500713.

[157] Santa-Maria M, Jeoh T. Molecular-Scale Investigations of Cellulose Microstructure during Enzymatic Hydrolysis. Biomacromolecules. 2010 Aug 9;11(8):2000-7.

[158] Thygesen LG, Hidayat BJ, Johansen KS, Felby C. Role of supramolecular cellulose structures in enzymatic hydrolysis of plant cell walls. Journal of Industrial Microbiology \& Biotechnology. 2010 Sep 18;38(8):975-83.

[159] Luterbacher JS, Walker LP, Moran-Mirabal JM. Observing and modeling BMCC degradation by commercial cellulase cocktails with fluorescently labeled Trichoderma reseii Cel7A through confocal microscopy. Biotechnology and Bioengineering. 2013 Jan;110(1):108-17. 